\title{
Separating Illumination and Surface Spectral from Multiple Color Signals
}

複数の反射光スペクトルを用いた 光源スペクトル・分光反射率分離

$$
\text { by }
$$

\section{Akifumi Ikari}

猪狩 壮文
A Master Thesis
修士論文

Submitted to the Graduate School of

Information Science and Technology

the University of Tokyo

of February, 2004

in Partial Fulfillment of the Requirements

for the Degree of Master of

Information Science and Technology

in Computer Science

Thesis Supervisor: Katsushi Ikeuchi 池内 克史

Professor of Computer Science 



\section{Acknowledgements}

I am thankful to Professor Katsushi Ikeuchi, Robby T. Tan, Daisuke Miyazaki,

Takaaki Harada and Rei Kawakami who had many precious opinions enjoyed in creating this paper. Moreover, I say gratitude also to you of the Ikeuchi laboratory who had you cooperate in order to advance research deeply, at this place. 


\begin{abstract}
A number of methods have been proposed to separate a color signal into its components: illumination spectral power distribution and surface spectral reflectance. Most of these methods usually use a minimization technique from solely a single color signal, which works in theoretical framework but is not effective for real data. The reason is it lacks the constraints necessary to make the iteration converge into correct separation. To resolve this problem, we proposed a minimization technique that, unlike the existing methods, uses multiple color signals. In our implementation, we introduce three different approaches: first, color signals obtained from two different surface reflectance lit by an identical illumination spectral power distribution; second, color signal from an identical surface reflectance lit by different illumination spectral power distributions; and third, color signals from identical surface reflectance but with different types of reflection components (diffuse and specular reflectance) lit by identical illumination spectral power distribution. Using multiple color signals can improve the robustness of the estimation, since we can obtain more constraints in the input data. And the experimental results on real spectral show the effectiveness of our method. In addition, practically we implement our method to deal with color signals of a scene taken using interference variable filter. The purpose is to obtain surface spectral reflectance and illumination spectral power distribution under some illumination light source.
\end{abstract}




\section{要旨}

反射光スペクトルをその構成成分である光源スペクトルと分光反射率に分離 する様々な手法が提案されてきた。それらの手法では主に単一の反射光スペ クトルを用いて誤差の最小化を行う手法が用いられる。しかしそれらは理論 的には正しい解が得られるが、実際のデータでは制約が不十分であるために 正しい解が得られない。そこで我々はそれらの問題を解決するために、今ま での単一の反射光スペクトルを使う手法を拡張して、複数の反射光スペクト ルを使う手法を提案する。ここで用いられる複数の反射光スペクトルは、 1. 同一光源下の異なった点のもの、2. 異なった光源下での同一の点のもの、3. 同一物体の同一光源下で内部反射している点と表面反射している点のもの、 のいずれかである。そしてこれらの複数の反射光スペクトルを用いることで 多くの制約が新たに加えられ、より精度の高い解を得ることができ、さらに 計算の安定性が向上した。さらに実際に様々な光源下で線形多波長フィルター を用いて多波長画像を取得し、その画像に対して分離を行い分光反射率画像 及び光源スペクトルを取得した。 


\section{Contents}

1 Introduction 1

2 Theoretical Background 4

2.1 Linear Basis Functions . . . . . . . . . . . . . . . . . 4

2.2 Separation Method . . . . . . . . . . . . . . . 7

3 Proposed method: Multiple Color Signals 9

3.1 Different Surface Reflectance Identical Illumination . . . . . . . . 9

3.1.1 Minimization of the approximation and measured value . 9

3.1.2 Minimization of the Difference of the Estimated Light Source of Multi Points . . . . . . . . . . . . . . 10

3.2 Identical Surface Reflectance Different Illumination . . . . . . . 12

3.2.1 Minimization of the approximation and measured value . 12

3.2.2 Minimization of the Difference of Estimated Reflectance of Multi Points . . . . . . . . . . . . . . . . . . 13

3.2.3 Multi pair under different illumination . . . . . . . . 15

3.3 Specular and Diffuse Points . . . . . . . . . . . . . . 16

3.3.1 Minimization of the approximation and measured value . 16

3.3.2 Minimization of Linear Combination of Specular and Diffuse Data and Approximated Illumination Distribution . . 18

4 Systems $\quad 21$

4.1 Equipments ........................ 21

4.2 Difference of Interference Filter and Spectral Line Scaner . . . . . 24

4.3 Perspective and Rotational . . . . . . . . . . . . . . 26

4.4 Conversion of Input Data . . . . . . . . . . . . . . . . . . . . 29

4.5 Conversion data obtaining . . . . . . . . . . . . 32 
5 Implementation

6 Experimental Results

7 Conclusion

49 


\section{List of Figures}

1 a. Judd's [11] three basis functions of illumination distribution.

b. Parkkinen's [16] four first basis functions of surface spectral reflectance. ..................... 6

2 Example of different surface, identical illumination . . . . . . . 11

3 Example of identical surface, different illumination . . . . . . . . 14

4 Example of a specular color signal and diffuse color signal . . . . 20

5 Spectrometer ......................... 22

6 Line spectral scaner . . . . . . . . . . . . . . 22

7 Interference filter and monochronic camera(SONY XC55) . . . 23

8 The range of penetration light of interference filter . . . . . . . 23

9 Parallel translation . . . . . . . . . . . . . . . . 28

10 Pan rotation . . . . . . . . . . . . . . . . 28

11 Distance from camera of parallel translation . . . . . . . . 31

12 Distance from camera of pan rotation . . . . . . . . 31

13 Measured real illumination distribution of outdoors and the result of separation algorithm using left: one point and right: multi points. 39

14 Measured real illumination distribution of incandescent light at $2800 \mathrm{~K}$ and the result of separation algorithm using left: one point and right: multi points. . . . . . . . . . . . . . . 39

15 Measured real illumination distribution of outdoors and result of separation algorithm with three, four, six and eight reflectance basis functions using all eighteen colors of the Macbeth Color Checker. 40

16 Measured real illumination distribution of outdoor and incandescent light at $2800 \mathrm{~K}$ and the result of the separation algorithm using identical surface(left:blue, right:green) reflectance under these illuminations. . . . . . . . . . . . . . . . . 41 
17 Measured real illumination distribution of outdoor and incandescent light at $2800 \mathrm{~K}$ and the result of the separation algorithm(25) using identical surface(left:blue, right:red) reflectance under these illuminations. . . . . . . . . . . . . . . . . 42

18 Measured real illumination distribution of left: halogen lamp right:outdoors, and the result of separation algorithm, Equation (39) and (40), using red, green, and blue diffuse-specular points. . . . . . . . . . 43

19 Measured real illumination distribution of left: halogen lamp and right:outdoor, and the result of separation algorithm, Equation (45) and (46), using red, green, and blue diffuse-specular points. . 44

20 Conversion function of pararell transformation using an algorithm $(64) \ldots \ldots \ldots \ldots \ldots \ldots$. . . . . . . . . . . . . . . . . . .

21 Scene separation result using algorithm (26). Take a scene moving a camera parallel translation under incandescent light at $2800 \mathrm{~K}$ and outdoor illumination, using red, green, blue point of Macbeth Color Checker. . . . . . . . . . . . . . . . . . . . . . . 47

22 Scene separation result using specular and diffuse point. Take a scene moving a monocronic camera pan rotated under outdoor illumination. . . . . . . . . . . . . . . . . 48 


\section{List of Tables}

1 The error value of one point algorithm and our algorithm(Section number), using all combination of eighteen color of Macbeth Color Checker ......................... 45 


\section{Introduction}

Light reflected from an object, usually called color signal is the product of two components: illumination spectral power distribution and surface spectral reflectance. To separate a color signal into its components is an important issue in computer vision and color science since, by separating them we can obtain the actual surface spectral reflectance without being affected by illumination spectral power distribution. Generally speaking, this kind of separation is part of a color constancy algorithm[8][5][7][6], which is commonly done in a three color channel (RGB) operation instead of in spectral operation.

In three color channels (RGB), various color constancy methods have been proposed. For instance, Finlayson et al. [5] showed that illumination change which causes the problem of color constancy can be turned into a crucial constraint to solve the problem of color constancy itself. Using a straight-line approximation model of illumination in diagonal matrix components space, they proposed an intersection approach of two pixels with the same surface color but lit by different illumination. Tan et al. [21] introduced a method focusing on highlighted regions that could be applied for both single and multi-colored surface.

While the aforementioned methods are applicable for separating in three color channel data, unfortunately, most of them cannot be applied to spectral (color signal) separation, since spectral data cannot be converted into chromaticity values (on which most methods are based) without losing its spectral information (metamerism problem). Hence, for color signals, a different technique that is suitable for is required.

Tominaga et al. [25] have shown that, by using the dichromatic reflectance model, illuminatioin distribution can obtained using both highlighted (specular) regions and diffuse regions of two different surface colors. By using this method highly performanced results are obtained. Marchant et al. [13] introduced spectral 
constancy under daylight, by assuming that illumination could be approximated with a blackbody radiator. This method can detect whether a surface has the same surface spectral reflectance, yet it is not intended to separate illumination spectral power distribution and surface spectral reflectance.

Ho et al. [10] showed that, by considering illumination spectral power distribution and surface spectral reflectance to be the sums of linear basis functions $[2,7,10,11]$, the color signal separation can be done by minimizing the square difference of a color signal and product of the sums of linear illumination basis functions and surface spectral reflectance basis functions. While theoretically it can separate a color signal into its components, a few problems exist. First, some parts of the separated signal in certain cases, become negative, which infringes on the physical reality of the spectral components since, in reality, those components are always positive. Second, in cases where the constraints are insufficient, the minimization algorithm could be trapped in the local minimum, thereby producing incorrect separation.

Chang et al. [1] improved the method of Ho et al. by putting additional constraints on the illumination and surface reflection components, as well as using a simulated annealing algorithm and a hit-and-run algorithm to increase the efficiency and stability. Their method gives a more robust result compared with that of Ho et al.; however, their separation still suffers from the same drawbacks as those of Ho et al's method. Their main problem is that a single color signal has such limited constraints that no current algorithm can avoid the trap of the local minimum.

In this paper, our goal is to describe how to separate color signals into illumination spectral power distribution and surface spectral reflectance components by giving more constraints in the input data, and separate a spectral images into illumination spectral power distribution and reflectance spectral images. We propose a minimization technique that, unlike the existing methods, uses multiple color 
signals. These multiple color signals can improve the robustness of the estimation because, by using them, we can obtain more constraints in the input data. In our implementation of using multiple color signals, we introduce three different approaches: first, color signals obtained from two different surface reflectance lit by identical illumination spectral power distribution; second, color signal from identical surface reflectance lit by different illumination spectral power distributions; and third, color signals from identical surface reflectance but different types of reflection components (diffuse and specular pixels) lit by identical illumination spectral power distribution. By using these three conditions of color signals, a better solution can be obtained and the stability of the separation increases.

To obtain a spectral images, Schechner[18] shows by using a interference filter and performing mosaicing algorithm[20][14][27][15], we can obtain spectral images. But by this approach we can't obtain spectral distribution exactly because of using gray world assumption. To obtain spectral distribution exactly, we have to obtain a conversion function that including camera parameter, filter parameter and distance for items. So we show how we obtain a conversion function correctly. And then, we perform separation algorithm to these spectral images.

The rest of the paper is organized as follows. In Section 2, we discuss the theoretical background of the proposed method. In Section 3, we explain our method of dealing with multiple color signals. The method to obtain a color signal is showed in Section 4. The implementation of our algorithm and the experimental results are provided in Section 5 and Section 6. And finally, in Section 7 we conclude our paper. 


\section{Theoretical Background}

\subsection{Linear Basis Functions}

A color signal spectrum is the product of illumination spectral power distribution (SPD) and surface spectral reflectance components. Mathematically, it is expressed as:

$$
I(\lambda)=E(\lambda) S(\lambda)
$$

where $I(\lambda)$ is the color signal at a wavelength $\lambda, E(\lambda)$ is the illumination spectral power distribution and $S(\lambda)$ is the surface spectral reflectance.

A number of researchers have asserted that the natural illumination can be approximated into a limited number of linear basis functions. Judd at el. [11] shows that illumination distribution of daylight and some indoor light sources can be approximated into a certain number of linear basis functions. They expressed the approximated illumination SPD as:

$$
E(\lambda) \simeq \sum_{i=1}^{m} e_{i} E_{i}(\lambda)
$$

where $E_{i}(\lambda)$ is the linear basis functions of illumination distribution and $e_{i}$ is the coefficient.

Furthermore, Judd et al. showed that only three linear basis functions are sufficient to express all natural illumination SPDs $(m=3)$. Slater et al. [19] argued that more sophisticated approximation needs eight linear basis functions. Yet, they also admitted that the first three basis functions dominantly cover all illumination SPDs. Ho et al. [10] and Chang et al. [1] used three basis functions in their estimation process. Fig.1.a shows the Judd's three illumination basis functions.

Similar to illumination SPDs, several researchers $[2,16]$ have shown that the surface spectral reflectance can be expressed in a number of linear basis functions: 


$$
S(\lambda) \simeq \sum_{j=1}^{n} s_{j} S_{j}(\lambda)
$$

where $S_{j}(\lambda)$ is the reflectance linear basis functions and $s_{j}$ is the coefficient.

Cohen et al. [2] determined his linear basis functions by investigating Munsell chips. Parkkinen et al. [16] examined various surface colors, concluding that eight basis functions could completely cover all of the existing surface color database. Moreover, they also showed that the first three basis functions cover $99 \%$ of the database. Fig.1.b shows the Parkkinen's four reflectance basis funtions. Ho et al. [10] and Chang et al. [1] used three basis functions for surface spectral reflectance. And it is natural if you increase a number of basis function, illumination SPD and surface spectral reflectance can reveail more exactly. 


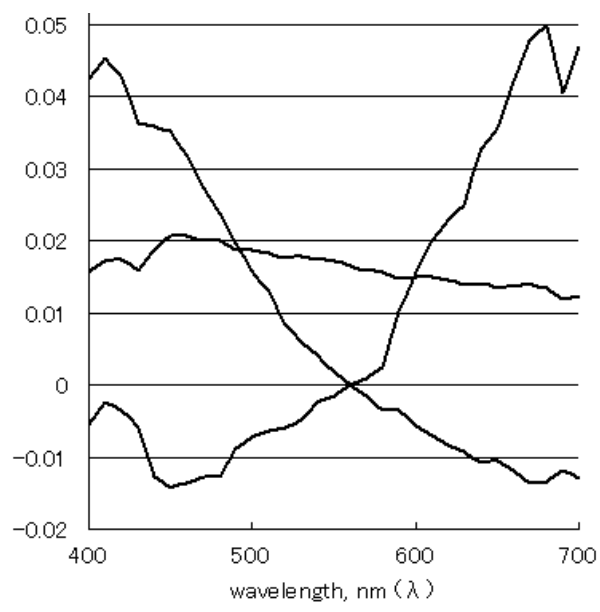

a.

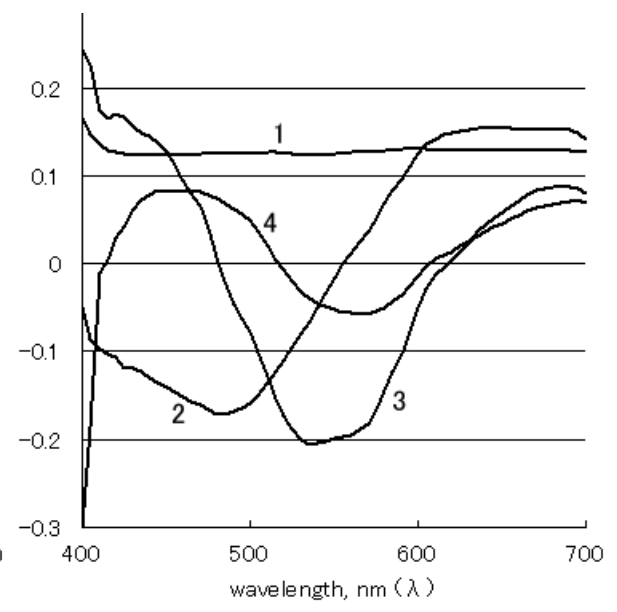

b.

Figure 1: a. Judd's [11] three basis functions of illumination distribution. b. Parkkinen's [16] four first basis functions of surface spectral reflectance. 


\subsection{Separation Method}

By plugging Equation (2) and (3) into Equation (1), a color signal can be approximated as:

$$
\begin{aligned}
I(\lambda) & =E(\lambda) S(\lambda) \\
& \simeq \sum_{i=1}^{m} e_{i} E_{i}(\lambda) \sum_{j=1}^{n} s_{j} S_{j}(\lambda)
\end{aligned}
$$

The Illumination SPD and surface spectral reflectance can be resolved using minimization technique;

$$
E R R O R=\sum_{\lambda}\left[\sum_{i=1}^{m} e_{i} E_{i}(\lambda) \sum_{j=1}^{n} s_{j} S_{j}(\lambda)-I(\lambda)\right]^{2}
$$

practically, the wavelength ranges over the visible spectrum from $400 \mathrm{~nm}$ to $700 \mathrm{~nm}$ with interval $5 \mathrm{~nm}$. By minimize the $E R R O R$ by changing a coeficients $e_{i}, s_{j}$, we can reveal a color signal and obtain a surface spectral reflectance and illumination SPD.

One of the problems using Equation (6) is that, in certain cases, the separation does not represent physical reality of illumination and surface spectral. Thus, to obtain more realistic results, Chang et al. [1] introduced additional constraints for illumination SPD and surface spectral reflectance. The constraints are expressed as:

$$
\begin{gathered}
I(\lambda) \leq \sum_{i=1}^{m} e_{i} E_{i}(\lambda) \leq \text { maxlimit } \\
0 \leq \sum_{j=1}^{n} s_{j} S_{j}(\lambda) \leq 1
\end{gathered}
$$

Constraint in Equation (7) means that the illumination SPD is always bigger than the input color signal. The upper limitation has no physical mean but it is 
important for computation. Equation (8) means surface spectral reflectance has values between zero and one.

Chang et al. [1] applied the constraints into the minimization algorithm of Equation (6) using hit-and-run algorithm and simulated annealing algorithm. 


\section{Proposed method: Multiple Color Signals}

\subsection{Different Surface Reflectance Identical Illumination}

\subsubsection{Minimization of the approximation and measured value}

To our knowledge, the existing methods consider the use of information from solely one single point. However, in the real world, each scene has an abundance of available points. Thus, instead of using a single color signal, we can derive benefit from this abundance of color signal types. Our first algorithm considers two types of color signals obtained from two different surface spectral reflectances illuminated by identical illumination SPD. In this case, the decomposition can be achieved by minimizing a sum of Equation (6) at each point under the constraint that the spectrum distribution of the illumination is common over the points, while their reflectance spectrums are different from each other. This constraint can be expressed as:

$$
\sum_{p=1}^{\text {points }} \sum_{\lambda}\left(\sum_{i=1}^{m} e_{i} E_{i}(\lambda) \sum_{j=1}^{n} s_{p, j} S_{j}(\lambda)-I_{p}(\lambda)\right)^{2}
$$

We use the constraint described in Equation (7) and the following equation:

$$
0 \leq \sum_{j=1}^{n} s_{p, j} S_{j}(\lambda) \leq 1
$$

The more spectrals are used, the more constraints can be obtained. Hence, this more constrained separation theoretically can produce a more accurate result. For efficient and stable decomposition, it is important to choose points with chromatically distributions as different as possible. 


\subsubsection{Minimization of the Difference of the Estimated Light Source of Multi Points}

When we consider a lot of point's color signals that illuminaiton is the same, the illumination distribution of each point can expressed as follows, using reflectance bsasis functions;

$$
\begin{aligned}
E(\lambda) & =\frac{I(\lambda)}{S(\lambda)} \\
& \simeq \frac{I_{1}(\lambda)}{\sum_{i=1}^{m} s_{1, i} S_{i}(\lambda)} \\
& \simeq \frac{I_{2}(\lambda)}{\sum_{i=1}^{m} s_{2, i} S_{i}(\lambda)}
\end{aligned}
$$

It is as follows when this formula is transformed.

$$
\begin{gathered}
\frac{I_{1}(\lambda)}{\sum_{i=1}^{m} s_{1, i} S_{i}(\lambda)} \simeq \frac{I_{2}(\lambda)}{\sum_{i=1}^{m} s_{2, i} S_{i}(\lambda)} \\
\frac{I_{1}(\lambda)}{\sum_{i=1}^{m} s_{1, i} S_{i}(\lambda)}-\frac{I_{2}(\lambda)}{\sum_{i=1}^{m} s_{2, i} S_{i}(\lambda)} \simeq 0
\end{gathered}
$$

So by minimizing the following equation, we can obtain the separated illuminaiton distribution and surface reflectance.

$$
\sum_{\lambda}\left(\frac{I_{1}(\lambda)}{\sum_{i=1}^{m} s_{1, i} S_{i}(\lambda)}-\frac{I_{2}(\lambda)}{\sum_{i=1}^{m} s_{2, i} S_{i}(\lambda)}\right)^{2}
$$




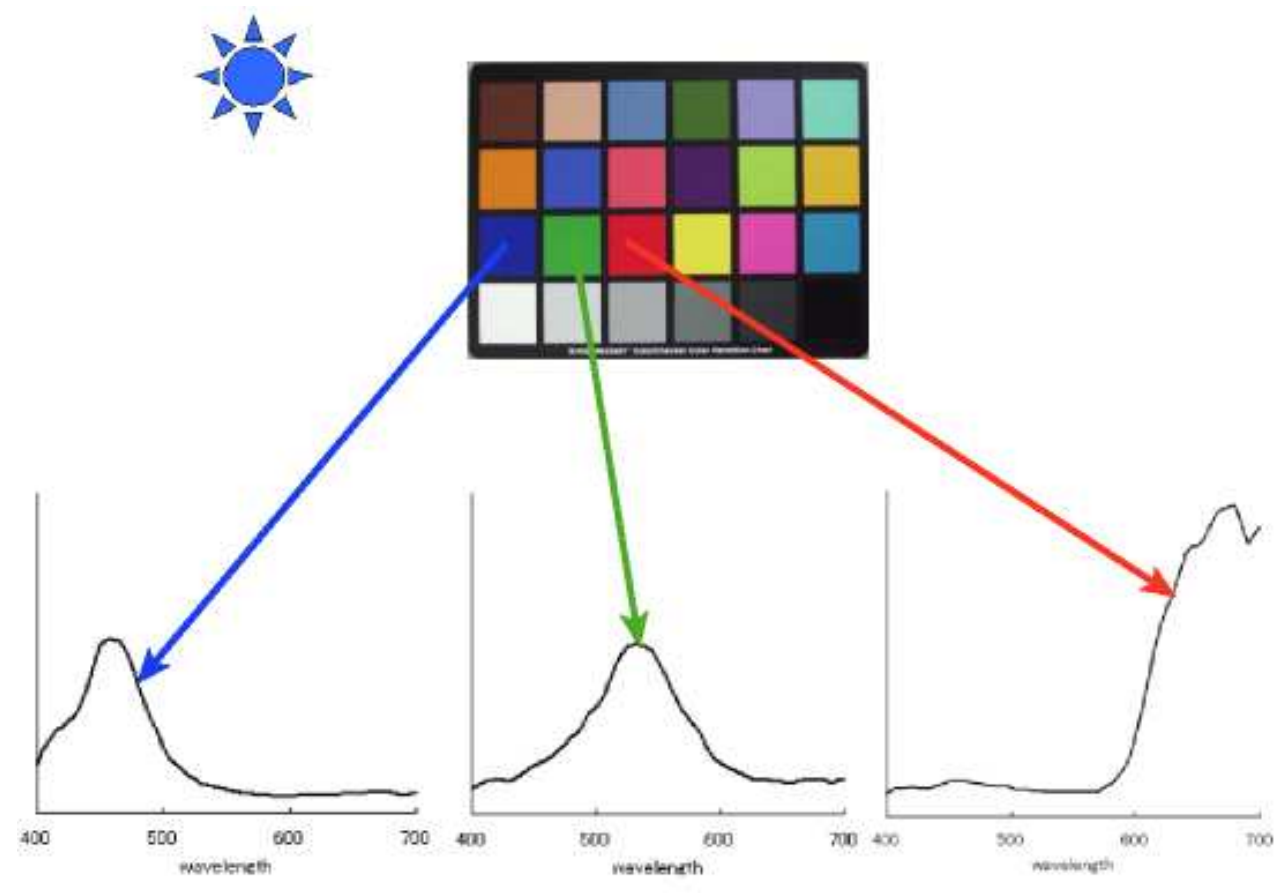

Figure 2: Example of different surface, identical illumination 


\subsection{Identical Surface Reflectance Different Illumination}

\subsubsection{Minimization of the approximation and measured value}

Another constraint can be obtained by considering multi spectrum distributions at the same surface spectral reflectance illuminated by different illumination SPD. We can resolve the separation problem by minimizing the sum of Equation (6) of each point under different illumination distributions:

$$
\sum_{p=1}^{\text {points }} \sum_{\lambda}\left(\sum_{i=1}^{m} e_{p, i} E_{i}(\lambda) \sum_{j=1}^{n} s_{j} S_{j}(\lambda)-I_{p}(\lambda)\right)^{2}
$$

and using the constraint described in Equation (8) and the following equation:

$$
I(\lambda) \leq \sum_{i=1}^{m} e_{p, i} E_{i}(\lambda) \leq \text { maxlimit }
$$

The problem of this approach is similar to that of subsection 3.1, but the constraints are different. In this approach, illumination constraints play a dominant role, which theoretically gives more constraints as compared with the approach in subsection 3.1. 


\subsubsection{Minimization of the Difference of Estimated Reflectance of Multi Points}

When we consider two color signals of identical point that illuminaiton is differect, the surface reflectance can expressed as follows using illumination basis functions.

$$
\begin{aligned}
S(\lambda) & =\frac{I(\lambda)}{E(\lambda)} \\
& \simeq \frac{I_{1}(\lambda)}{\sum_{i=1}^{m} e_{1, i} E_{i}(\lambda)} \\
& \simeq \frac{I_{2}(\lambda)}{\sum_{i=1}^{m} e_{2, i} E_{i}(\lambda)}
\end{aligned}
$$

It is as follows when this formula is transformed.

$$
\begin{aligned}
& \frac{I_{1}(\lambda)}{\sum_{i=1}^{m} e_{1, i} E_{i}(\lambda)} \simeq \frac{I_{2}(\lambda)}{\sum_{i=1}^{m} e_{2, i} E_{i}(\lambda)} \\
& I_{1}(\lambda) \sum_{i=1}^{m} e_{2, i} E_{i}(\lambda) \simeq I_{2}(\lambda) \sum_{i=1}^{m} e_{1, i} E_{i}(\lambda) \\
& I_{1}(\lambda) \sum_{i=1}^{m} e_{2, i} E_{i}(\lambda)-I_{2}(\lambda) \sum_{i=1}^{m} e_{1, i} E_{i}(\lambda) \simeq 0
\end{aligned}
$$

So by minimizing the following equation, we can obtain the separated illuminaiton distribution and surface reflectance.

$$
\sum_{\lambda}\left(I_{1}(\lambda) \sum_{i=1}^{m} e_{2, i} E_{i}(\lambda)-I_{2}(\lambda) \sum_{i=1}^{m} e_{1, i} E_{i}(\lambda)\right)^{2}
$$




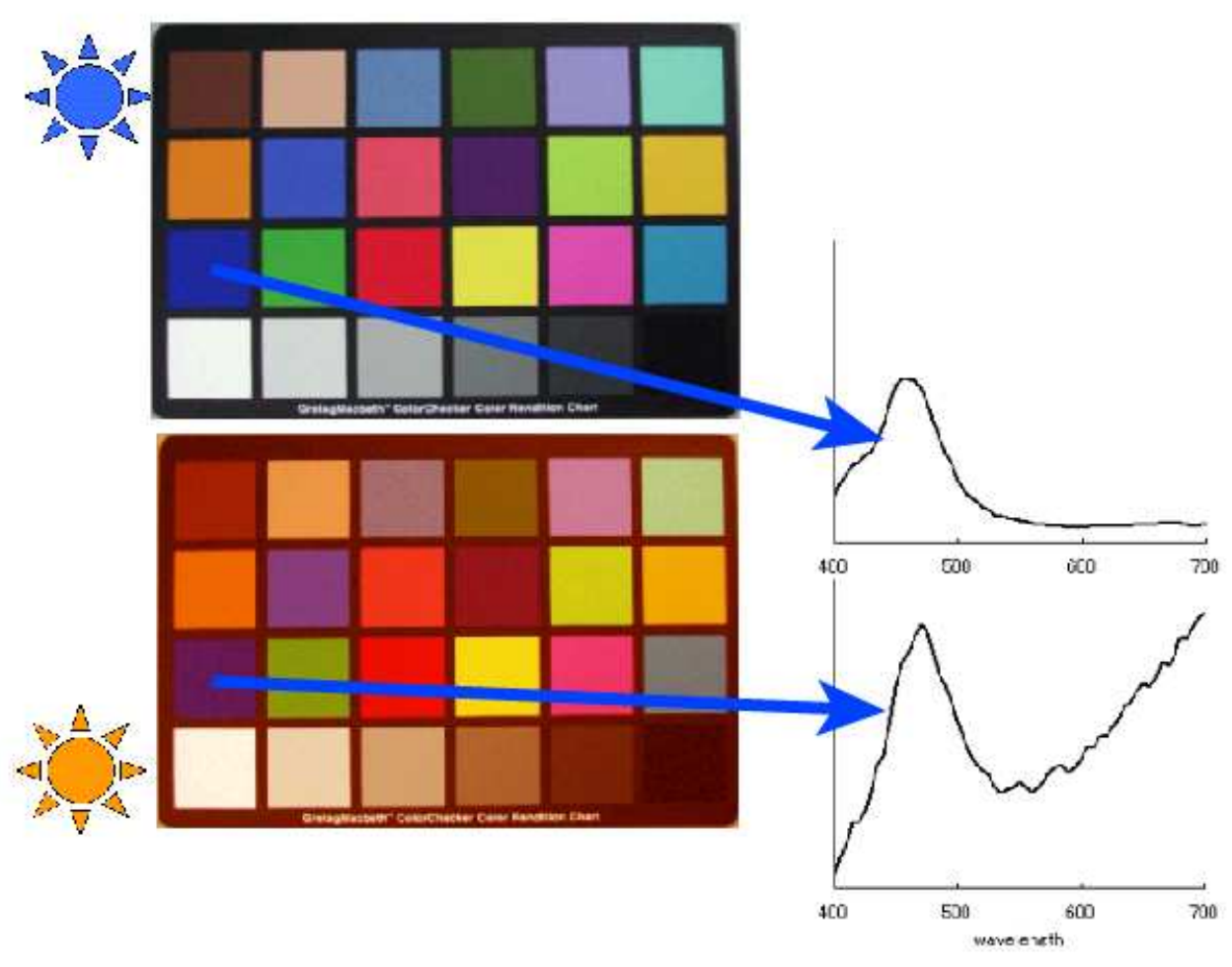

Figure 3: Example of identical surface, different illumination 


\subsubsection{Multi pair under different illumination}

Then, consider a case, using multi pairs of identical point's color signal under different illumination. In this case, illumination spectral power distribution and surface spectral reflectance reveail as follows:

$\sum_{p=1}^{\text {points }} \sum_{\lambda}\left(\left(\sum_{i=1}^{m} e_{1, i} E_{i}(\lambda) \sum_{j=1}^{n} s_{p, j} S_{j}(\lambda)-I_{p, 1}(\lambda)\right)^{2}+\left(\sum_{i=1}^{m} e_{2, i} E_{i}(\lambda) \sum_{j=1}^{n} s_{p, j} S_{j}(\lambda)-I_{p, 2}(\lambda)\right)^{2}\right)$

here, $(p, 1)$ and $(p, 2)$ is the pair of same items under different light source.

If we use this folmula, it has a strong constraint. 


\subsection{Specular and Diffuse Points}

\subsubsection{Minimization of the approximation and measured value}

In the real world, there are many objects with dielectric inhomogeneous material. This kind of material exhibits specular and diffuse reflections as described in the dichromatic reflection model. By using this material as target objects, we consider that we can gain benefit from the color signals of specular and diffuse points illuminated by the same illumination SPD. In this subsection, we will explain the algorithm and show the relationship between diffuse and specular points.

In the dichromatic reflectance model, the observed spectrum distribution at a specular point can be expressed as follows:

$$
I(\lambda) \simeq m_{s}(i, e, g) E(\lambda) S_{s}(\lambda)+m_{b}(i, e, g) E(\lambda) S_{b}(\lambda)
$$

where $m_{s}, m_{b}$ are constant scalars, $S_{s}(\lambda)$ is the specular spectral reflectance function, and $S_{b}(\lambda)$ is the diffuse spectral reflectance function. $S_{b}$ can be approximated using:

$$
S_{b}(\lambda) \simeq \sum_{j=1}^{n} s_{j} S_{j}(\lambda)
$$

the geometric factors $\left(m_{s}\right.$ and $\left.m_{d}\right)$ can also be rewritten as:

$$
\begin{aligned}
& m_{s}(i, e, g)=C_{s} \\
& m_{b}(i, e, g)=C_{b}
\end{aligned}
$$

We can assume that the specular spectrum reflectance function is identical to the spectrum distribution of the incident light for most dielectric inhomogenous objects. Lee et al. [12] named this assumption the neutral interface reflection (NIR) assumption. Using this NIR assumption, we can set $S_{s}(\lambda)$ as a constant.

In this paper, Parkkinen et al. [16] showed the first basis function, $S_{1}$ can be approximated to be a constant (Fig.1.b, line 1), and independent of $\lambda$ :

$$
S_{s}(\lambda) \simeq k S_{1}(\lambda) \simeq \text { Constant }
$$


where $k$ is a constant scalar. then

$$
\begin{aligned}
I(\lambda) & \simeq C_{s} E(\lambda) S_{s}(\lambda)+C_{b} E(\lambda) S_{b}(\lambda) \\
& \simeq C_{s} E(\lambda) k S_{1}(\lambda)+C_{b} E(\lambda) \sum_{j=1}^{n} s_{j} S_{j}(\lambda) \\
& =E(\lambda) \sum_{j=1}^{n} s_{1, j} S_{j}(\lambda)
\end{aligned}
$$

A diffuse point on the same surface spectral reflectance has the following spectrum distribution:

$$
\begin{aligned}
I^{\prime}(\lambda) & \simeq C_{b}^{\prime} E(\lambda) S_{b}(\lambda) \\
& \simeq C_{b}^{\prime} E(\lambda) \sum_{j=1}^{n} s_{j} S_{j}(\lambda) \\
& =E(\lambda) \sum_{j=1}^{n} s_{2, j} S_{j}(\lambda)
\end{aligned}
$$

From Equation (34) and Equation (37) the relation between specular and diffuse point are expressed as:

$$
s_{1,2}: s_{1, j}=s_{2,2}: s_{2, j}(j=3 \sim n)
$$

Using this constraint, we can set up a more robust separation algorithm than those in the previous subsection 3.1.

In order to use the hit-and-run algorithm, we rewrite Equation (35) and Equation (32) into the following equation:

$$
\begin{gathered}
\sum_{p=1}^{\text {points }} \sum_{\lambda}\left(\alpha_{p} \sum_{i=1}^{m} e_{i} E_{i}(\lambda) \sum_{j=1}^{n} s_{p, j} S_{j}(\lambda)-I(\lambda)\right)^{2} \\
s_{1, j}=s_{2, j}(j=2 \sim n)
\end{gathered}
$$

where $\alpha_{p}$ is a new variable. Therefore, it can generate a new state efficiently using the hit-and-run algorithm. 


\subsubsection{Minimization of Linear Combination of Specular and Diffuse Data and Approximated Illumination Distribution}

As another way to obtain illumination distribution, from Equation (34) and Equation (37), illumination distribution can be expressed as follows:

$$
C_{b}^{\prime} I(\lambda)-C_{b} I^{\prime}(\lambda)=C_{b}^{\prime} C_{s} E(\lambda) S_{s}(\lambda)
$$

Then, by dividing Equation (41) by $C_{b}^{\prime}$, we can obtain the following variables:

$$
\begin{gathered}
q_{1}=\frac{C_{b}}{C_{b}^{\prime}} \\
q_{2}=C_{s} S_{s}(\lambda)
\end{gathered}
$$

consequently,

$$
\begin{aligned}
I(\lambda)-q_{1} I^{\prime}(\lambda) & =q_{2} E(\lambda) \simeq q_{2} \sum_{i=1}^{m} e_{i} E_{i}(\lambda) \\
& =\sum_{i=1}^{m} e_{i}^{\prime \prime} E_{i}(\lambda)
\end{aligned}
$$

We resolve (45) by minimizing the following equations;

$$
\sum_{\lambda}\left(I(\lambda)-q_{1} I^{\prime}(\lambda)-\sum_{i=1}^{m} e_{i}^{\prime \prime} E_{i}(\lambda)\right)
$$

We also use constraint described in Equation (7) and the following equation:

$$
0 \leq q_{1} \leq \frac{I(\lambda)}{I^{\prime}(\lambda)}
$$

By minimizing this equation changing a value of coefficient $q_{1}, e_{i}^{\prime \prime}$, we can obtain accurate illumination SPD. This theoretical analysis confirms the results of a number of researchers in color constancy, that is, specularity give a significant clue to 
estimate illumination color. Furthmore, (45) can expressed as linear equation as follows;

$$
\left(\begin{array}{llll}
E_{1}(\lambda) & \ldots & E_{m}(\lambda) & I^{\prime}(\lambda)
\end{array}\right)\left(\begin{array}{c}
e_{1} \\
\vdots \\
e_{m} \\
q_{1}
\end{array}\right)=I(\lambda)
$$

By this method, stability and calculation speed is increased. 


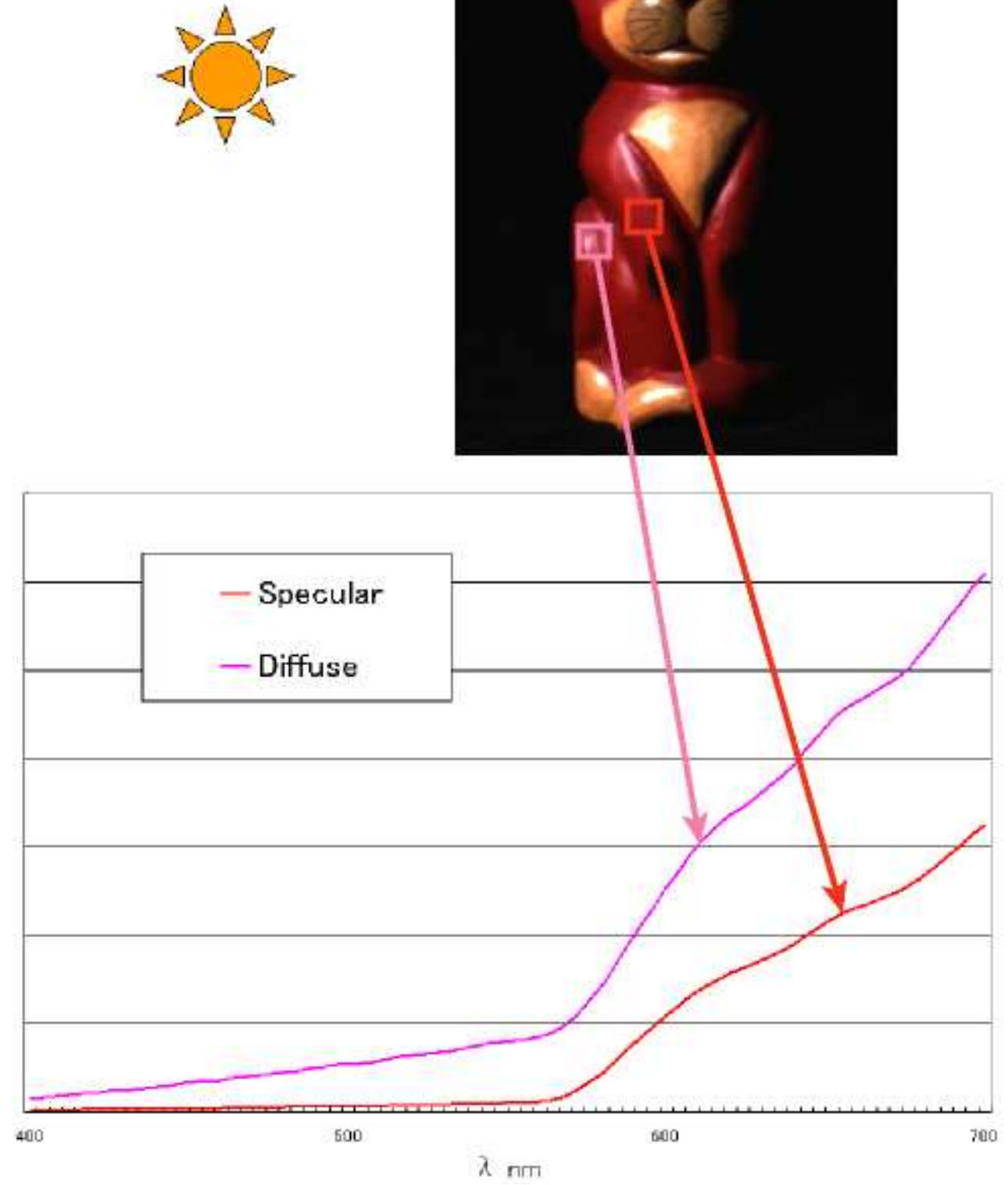

Figure 4: Example of a specular color signal and diffuse color signal 


\section{Systems}

\subsection{Equipments}

Spectrometer To obtain a spectral color signal, it is easy to use spectrometer(Fig. 5). Spectrometer can exactly measure a point's spectral distribution. But one of the problem is that because it measure a point's color signal, it is difficult to obtain spectral images.

Line Spectral Scaner To obtain multi spectral images, a unique system is needed because of a number of camera color sensor is so little. As one of the system, the way to use spectral line scaner(Fig.6) is exist. Line spectral scaner is attached a monochronic camera. By using the line scaner, we can obtain a line spectral image by image. So if we exactly know the moving motion of the camera and move slowly making a width between lines be small enough, multi spectral images are obtained.

Interference Filter As the another way to easily obtain a multi spectral images, Yoav [18] showed by using a interfelence filter(Fig.7) and monochronic camera, we can obtain spectral images. It is so good because we only need the interference filter and monochronic camera. Interference filter is a prism filter that the wavelength of penetration light crosses filter is change across the filter in visible light from 700nm to 400nm(Fig.8). Then, we shows process of obtaining spectral images. First, attached interference filter to an 8-bit monochrome camera, and then we take a scene moving the camera continuously by pan rotation or perspectively(4.3), and mosaic[20][14][27][15] these images using smoothing algorithm and edge detection algorithm. Then convert(4.4) the obtained data of luminosity value of each points to a spectral data. And then we can easily get the multi spectral images. 


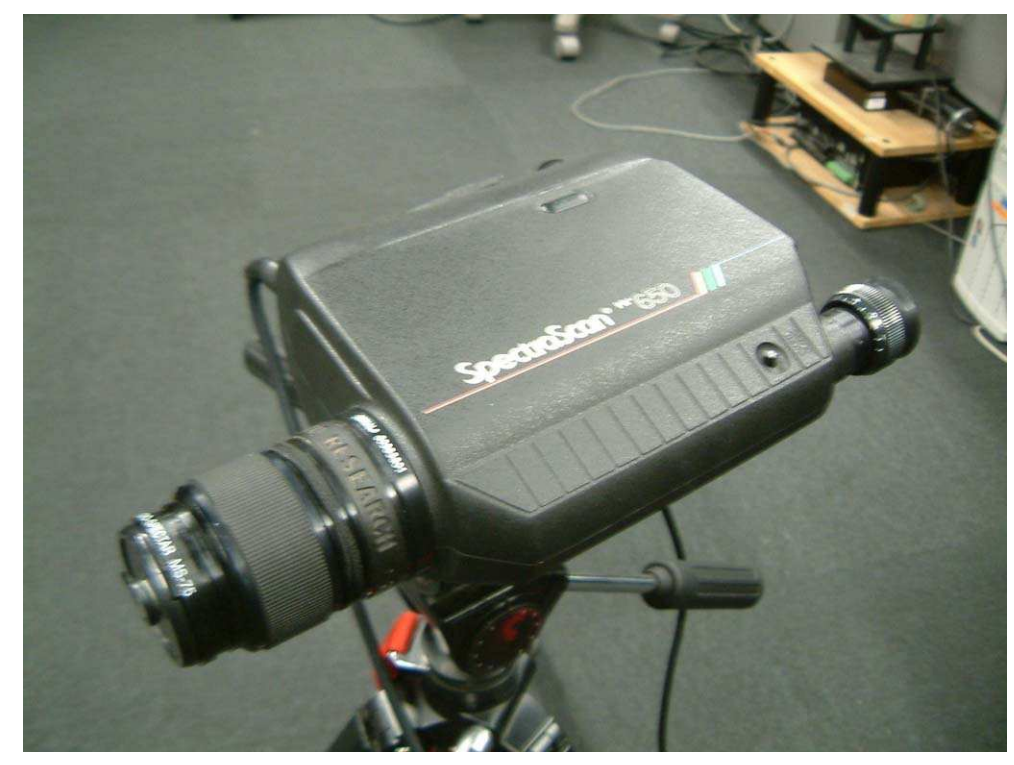

Figure 5: Spectrometer

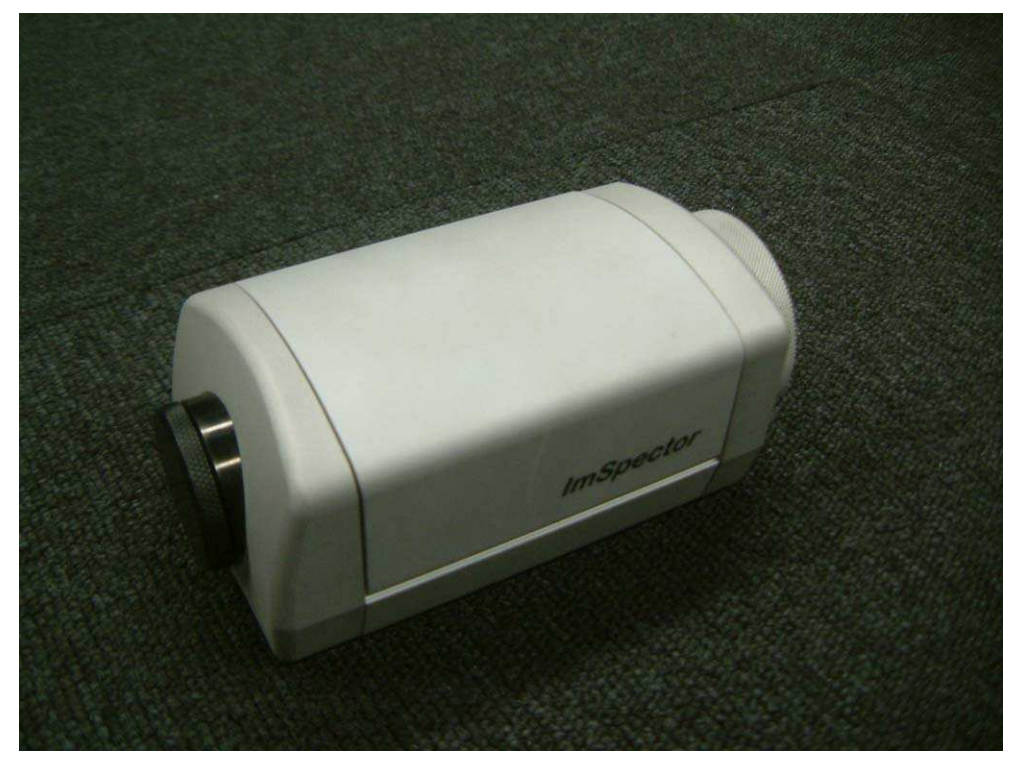

Figure 6: Line spectral scaner 


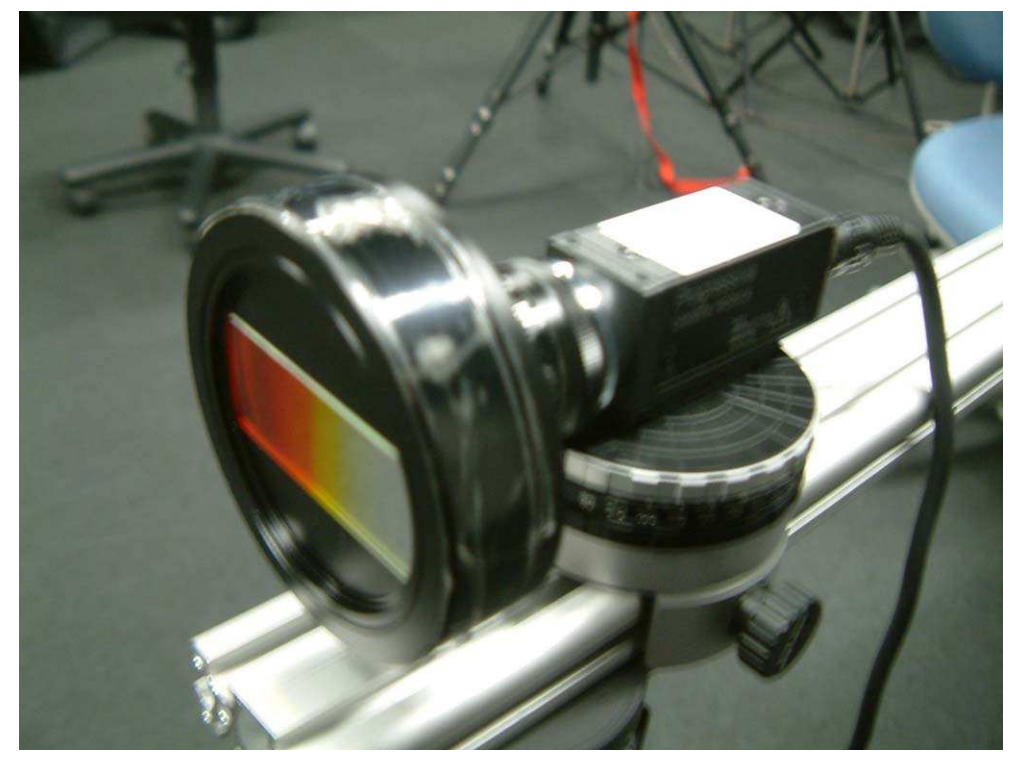

Figure 7: Interference filter and monochronic camera(SONY XC55)

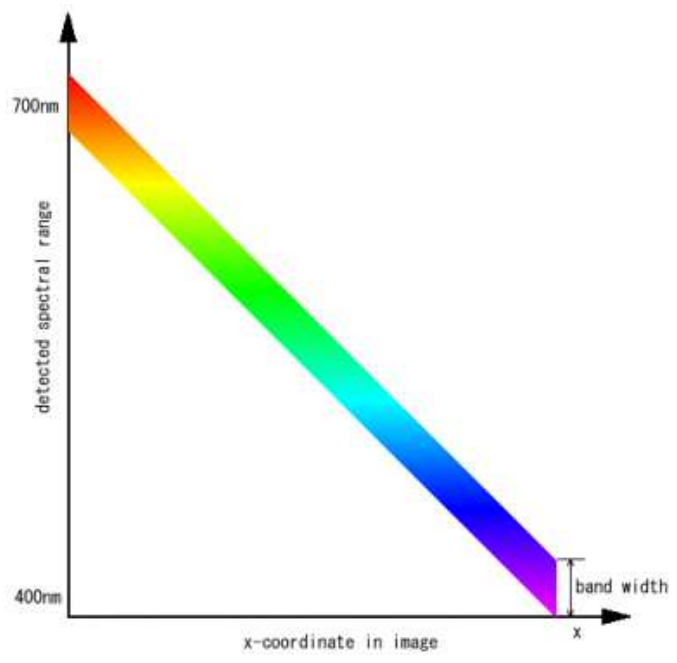

Figure 8: The range of penetration light of interference filter 


\subsection{Difference of Interference Filter and Spectral Line Scaner}

To take a spectral, we need to use interference filter or spectral line scaner. These has merits and demerits. So here, we show these merits and demerits.

- Interference filter

- Advantage

* Can control a density of spectral color signal.

* Camera motion can obtained from continuously taked images.

- Disadvantage

* Multi images are needed to get a point's spectral power distribution.

* Mosaicing is needed.

* Azimuth difference arises except when a camera pan rotate.

* Can't take a specular spectral easily, especially when a camera translate parallely it is difficult to obtain a specular spectral because a point of specular is move depend on the camera's position.

- Prismic filter

- Advantage

* Can take a line spectral distribution only using one image.

* Easily take a specular spectral.

* No azimuth difference.

- Disadvantage 
* Only can control a width between lines. To take a density spectral image, it is necessary to move so slow or increase a photography speed(fps)

* It is necessary to know a camera's motion from some data, because these image has no infomation

For our experiments, it's necessary to take a scene spectrum. And if we use a spectral line scaner, it's needed to move so slowly and to know a camera motion, and it is so difficult. So we use interference filtler although there are some disadvantage. 


\subsection{Perspective and Rotational}

When we use interference filter, it is neccesarry to take an object at a lot of sufficient position $(\mathrm{x})$ of images. So we need to move a monocronic camera that filter attached, and continuously took images. Then, as the camera motion to take images continuously, we use two types of motions. One of the motion is parallel translation, turning a camera perpendicularly to a photography plane, and photoing a camera, while fixing distance with a plane(Fig.9). And another motion is pan rotation, rotating a camera at the camera's camera center, and taking a scene continuously(Fig.10)

These two motion has advantage and disadvantage, so we shows these advantage and disadvantage.

- Parallel Translation

- Advantage

* Data can be much obtained continuously.(Camera can move long distance.)

- Disadvantage

* Can’t obtain a specular spectral data.

* Azimuth difference arises.

- Pan Rotation

- Advantage

* Can obtain a specular data (When mosaic image exactly).

* No azimuth difference.

- Disadvantage 
* Only a spectral image from a camera's position(Camera can't move, only can do a 360 degree rotation.)

To use a specular and diffuse color signal using interference filter, it is necessary to use pan rotation. And it is necessary to mosaic continuous images exactly, because specular point is required at the exact position, and if the position is shift a little, specular spectral has greatly large noises. So if we need a continuous images, like a town spectral images, or use datas that have little azimuth difference, it is better to use pallarell translation. And if we need a specular data or use datas that have largel azimuth difference, it is better to use pan translation. So it is necessary to use them properly. 


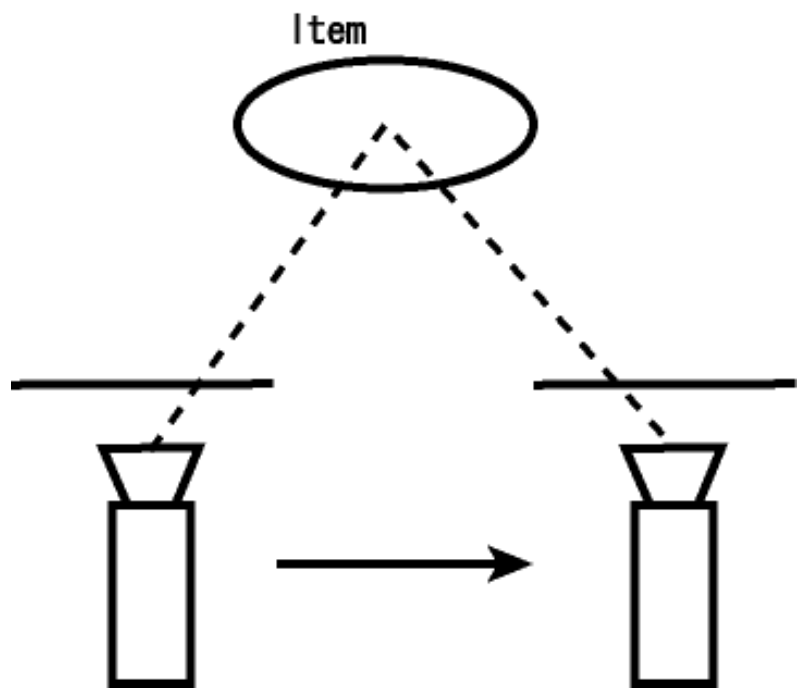

Figure 9: Parallel translation

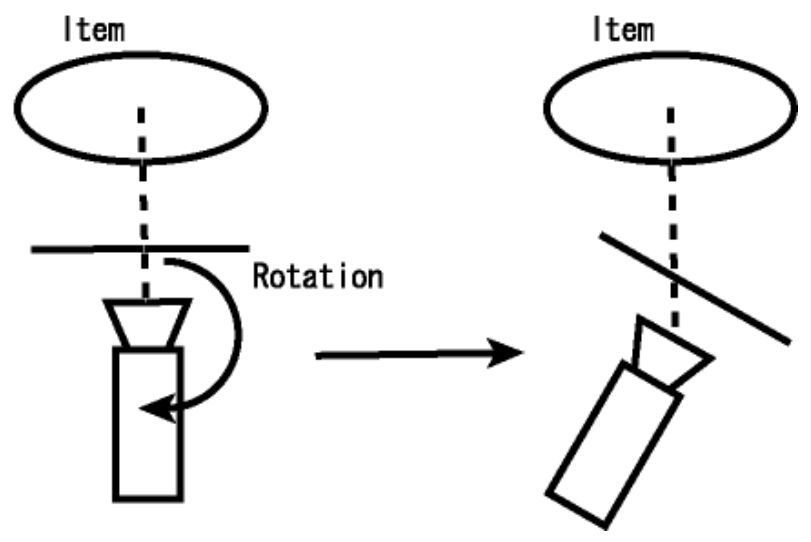

Figure 10: Pan rotation 


\subsection{Conversion of Input Data}

When we take a scene with a camera which interference filter attached, the mosaiced data is expressed as follow.

$$
I(x, y)=E\left(\lambda, x^{\prime}, y^{\prime}\right) S\left(\lambda, x^{\prime}, y^{\prime}\right) F(\lambda) C(\lambda) D\left(x^{\prime}, y^{\prime}\right)
$$

here $E$ is illumination distribution at the position $\left(x^{\prime}, y^{\prime}\right), S$ is surface reflectance, $F$ is transmissivity of interference filter, $C$ is a variation of camera sensor's reaction and $D$ is a distance function from a camera to the items position. So real spectral distribution is expressed as follows.

$$
\begin{aligned}
\text { Realspectral }(\lambda, y) & =E\left(\lambda, x^{\prime}, y^{\prime}\right) S\left(\lambda, x^{\prime}, y^{\prime}\right) \\
& =\frac{I(x, y)}{F(\lambda) C(\lambda) D\left(x^{\prime}, y^{\prime}\right)}
\end{aligned}
$$

\section{Pan Rotation}

When we took a scene rotating a camera at it's camera center, the distance from a camera center to an object is the same, no matter which direction camera has turned.

$$
D\left(x^{\prime}, y^{\prime}\right)=\text { Constant }
$$

So to get a spectral distritution, we need to get $H_{p a n}(\lambda)$. It reveals as follows.

$$
\begin{gathered}
H_{\text {pan }}(\lambda)=\frac{1}{F(\lambda) C(\lambda)} \\
\text { Realspectral }(\lambda, y)=\frac{I(\lambda, y)}{H_{\text {pan }}(\lambda)}
\end{gathered}
$$


2.Parallel translation

When we took a scene moving a camera parallel translation rightangled in the photography direction, the distance from a camera is different depends on a camera posistion. And the distance is relate on a image's position(x). It expressed as follows;

$$
\operatorname{Distance}\left(x^{\prime}, y^{\prime}\right)=\left(\frac{D_{\text {center }}}{\cos \theta}\right)^{2}
$$

Then, becuase of $\cos \theta$ depend on $\lambda$ it reveail as follows:

$$
\operatorname{Distance}\left(x^{\prime}, y^{\prime}\right)=\left(D_{\text {center }} \alpha(\lambda)\right)^{2}
$$

here, $D_{\text {center }}$ is the distance when item is taken at a center of image and $\theta$ is the direction of the item. So to get a spectral power distritution from mosaicing data, we need to get $H_{\text {parallel }(\lambda)}$. It reveals as follows.

$$
\begin{gathered}
H_{\text {parallel }(\lambda)}=\frac{1}{F(\lambda) C(\lambda)\left(D_{\text {center }} \alpha(\lambda)\right)^{2}} \\
\text { Realspectral }(\lambda, y)=\frac{I(\lambda, y)}{H_{\text {parallel }}(\lambda)}
\end{gathered}
$$

The function rectified from a mosaicing data to real spectral distribution is different between pan rotation and parallel translation. So it is necessary to make a different conversion function each. 


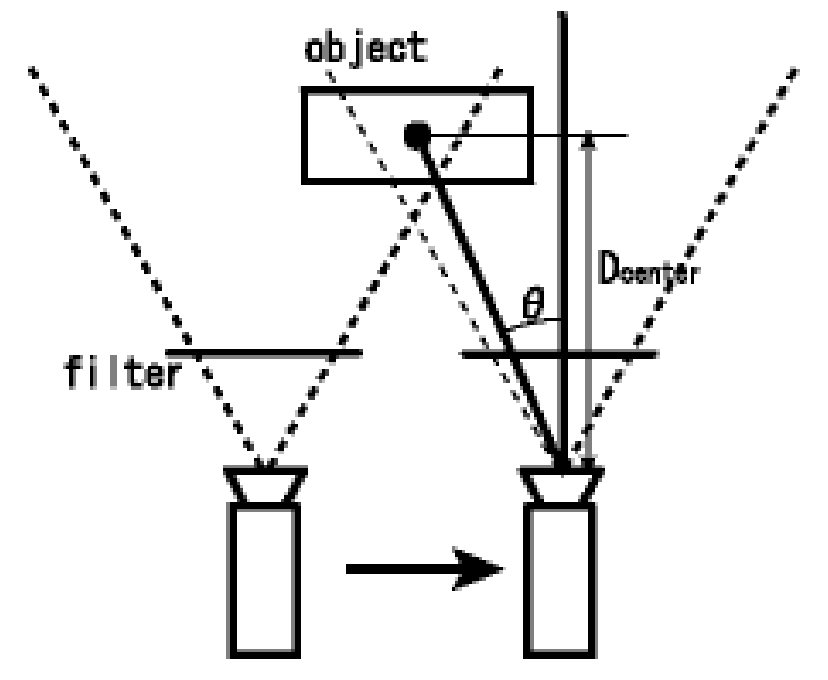

Figure 11: Distance from camera of parallel translation

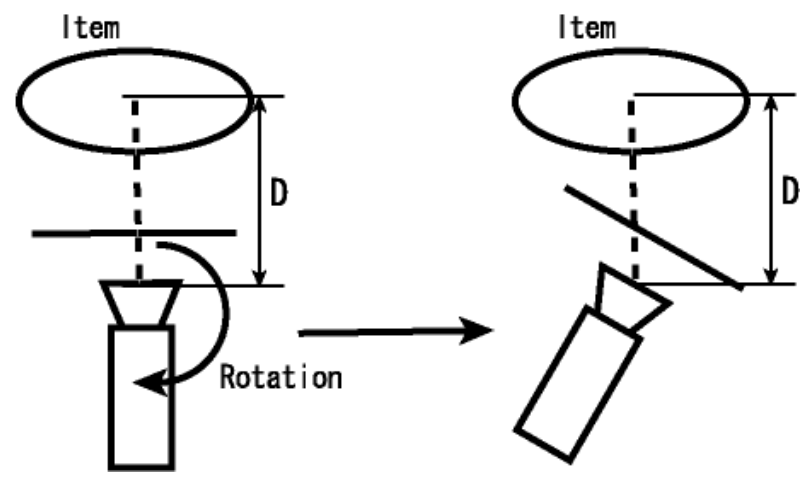

Figure 12: Distance from camera of pan rotation 


\subsection{Conversion data obtaining}

Compensation data is expressed with the quotient of the data directly obtained using spectrum meter, and the data which obtained from mosaiced data which obtained using a camera with a interference filter.

$$
H(\lambda)=\frac{E s p(\lambda)}{\operatorname{Evf}(\lambda)}
$$

here, $H(\lambda)$ is a compensation data, $\operatorname{Esp}(\lambda)$ is a data which obtained using spectrometer and $\operatorname{Evf}(\lambda)$ is a data which obtained mosaicing images which obtained using interfelence filter.

Then, when we get the compensation data, it is not sufficient using only one data because of noise and so on. So we use a lot of data for obtaining compensation data. In our experiments we use eighteen color of Macbeth Color Checker. We took these color under blue illumination with spectrometer and a camera with interference filter. And we mosaic camera captured images and compare with spectrometers image.

The compensation data is obtained by minimization algorithm. It expressed as following folmula;

$$
\sum_{p=1}^{18} \sum_{\lambda}\left(E s p_{p}(\lambda)-H(\lambda) * E v f_{p}(\lambda)\right)^{2}
$$

then, consider as follow;

$$
\begin{gathered}
\alpha(\lambda)=\sum_{p=1}^{18}\left(E v f_{p}(\lambda)\right)^{2} \\
\beta(\lambda)=-2 \sum_{p=1}^{18} \operatorname{Esp}_{p}(\lambda) E v f_{p}(\lambda) \\
\gamma(\lambda)=\sum_{p=1}^{18}\left(E s p_{p}(\lambda)\right)^{2}
\end{gathered}
$$


From (60)(61)(62), we can reveal (59) as follows;

$$
\begin{array}{r}
\alpha(\lambda) H(\lambda)^{2}+\beta(\lambda) H(\lambda)+\gamma(\lambda) \\
=\alpha(\lambda)\left(H(\lambda)+\frac{\beta(\lambda)}{2 \alpha(\lambda)}\right)^{2}+\gamma(\lambda)-\frac{\beta(\lambda)^{2}}{4 \alpha(\lambda)}
\end{array}
$$

So a compansation data that minimize (64) is express as follows;

$$
H(\lambda)=-\frac{\beta(\lambda)}{2 \alpha(\lambda)}
$$

We do these processing for pan rotation data and parallel translation data each. 


\section{Implementation}

Our implementation is based on the simulated annealing algorithm with a hit-andrun algorithm [1]. Using the simulated annealing algorithm enables us to avoid local minima in solving a non-linear optimization problem. While using hit-andrun algorithm, we can obtain a good performance in searching the interior point as a new state.

The simulated annealing algorithm is expressed as follows.

1. Gave an initial state for variable $\vec{u}=\left(e_{i}, s_{j}, \alpha\right)$ these are feasible.

2. Generate a new state $\overrightarrow{u^{\prime \prime}}$ using hit-and-run algorithms.

3. IF $\Delta F=F(\vec{u})-F\left(\overrightarrow{u^{\prime \prime}}\right)<0$ or $e^{-\Delta F / T_{e}(t)}>\operatorname{RANDOM}[0,1]$ THEN $\vec{u}=\overrightarrow{u^{\prime \prime}}$ and go to 2 .

ELSE go to 2.

Do these approach some times or until $F(\vec{u})$ becomes so small. And some times make $t=t+1$. Obtained $\vec{u}$ is a value which make $F(\vec{u})$ mostly small. Here, $F$ is evaluation function and $T_{e}(t)$ is called cooling function and we use $T_{e}(T)=$ Const $/(1+t)^{2}$. 
Hit and run alogrithm is used to make good performance to search interior point as a new state. It is useful if the space is convex. The process is as follows.

1. Given an interior point $\vec{u}$

2. Generate a vector $\overrightarrow{u^{\prime}}$ with equal probability.(randomly

3. Do next calculation.

$$
\begin{aligned}
k_{1, \lambda} & =\frac{\operatorname{maxlim}-\sum_{i=1}^{m} e_{i} E_{i}(\lambda)}{\sum_{i=1}^{m} e_{i}^{\prime} E_{i}(\lambda)}, k_{2, \lambda}=\frac{-\sum_{i=1}^{m} e_{i} E_{i}(\lambda)}{\sum_{i=1}^{n} e_{i}^{\prime} E_{i}(\lambda)} \\
k_{3, \lambda} & =\frac{1-\sum_{j=1}^{n} s_{j} S_{j}(\lambda)}{\sum_{j=1}^{n} s_{j}^{\prime} S_{j}(\lambda)}, k_{4, \lambda}=\frac{-\sum_{j=1}^{n} s_{j} S_{j}(\lambda)}{\sum_{j=1}^{n} s_{j}^{\prime} S_{j}(\lambda)} \\
r^{+} & =\min _{1 \leq k \leq 4, \lambda} K_{k, \lambda} \\
r^{-} & =\max _{1 \leq k \leq 4, \lambda} K_{k, \lambda} \\
\text { 4. } \overrightarrow{u^{\prime \prime}} & =\vec{u}+\left[r^{-}+\operatorname{RANDOM}[0,1]\left(r^{+}-r^{-}\right)\right] \overrightarrow{u^{\prime}}
\end{aligned}
$$

The $\overrightarrow{u^{\prime \prime}}$ is the output data. And use the $\overrightarrow{u^{\prime \prime}}$ in simmulated annealing as a new state. 


\section{Experimental Results}

To confirm the performance of separation algorithm, we perform an experiment using some samples. We measured a color signal pectrum using the Spectrascan PR650.By using Spectrascan PR650, we can measured a color signal from 380nm to $780 \mathrm{~nm}$ by $4 \mathrm{~nm}$ at the maximum, and we use a color signal from $400 \mathrm{~nm}$ to $700 \mathrm{~nm}$ by $5 \mathrm{~nm}$. We use Macbeth Color Checker as our target object. For the basis functions, we used Judd's [11] three illumination basis functions and Parkkinen's [16] reflectance basis functions.

Fig.13 shows the real illumination distribution of outdoors, and estimated illumination distribution with a separation algorithm under three reflectance basis functions, using one, two and three points of the Macbeth Color Checker's red, green and blue colors, respectively. Fig.14 shows the real illumination distribution of incandescent light at $2800 \mathrm{~K}$ and the estimated illumination distribution with the separation algorithm, under three reflectance basis functions, using one, two and three points of the Macbeth Color Checker's red, green and blue colors, respectively. As shown in Fig.14, if we use incandescent light, relatively good separation results are obtained utilizing the multi color signals algorithms with the red and blue, or the red and green and blue point's spectrals.

Fig.15 shows the real illumination distribution of outdoors, and the estimated illumination distribution using three, four, six, and eight reflectance basis functions, using eighteen point's spectral of the Macbeth Color Checker.

Fig.16 shows the real illumination distribution of outdoor and incandescent light at $2800 \mathrm{~K}$ and the estimated illumination distribution with the separation algorithm using blue and green surfaces under these illuminations. In this experiment, we use eight reflectance basis functions. As shown in Fig.16, if we use a blue point lit by incandesent light at $2800 \mathrm{~K}$ and outdoor illumination, we obtain a good result. 
Fig.17 shows the real illumination distribution of outdoor and incandescent light at $2800 \mathrm{~K}$ and the estimated illumination distribution with the separration algorithm (25) using red and blue surface each under these illuminations. As shown in Fig.17, if we use a red point lit by incandescent light at $2800 \mathrm{~K}$ and outdoor illumination, we have a especially good result.

Fig.18 shows the real illumination distribution of outdoor and incandescent light at $2800 \mathrm{~K}$ and the estimated illumination distributions using the diffuse and specular separation algorithm, using red, green, and blue points' diffuse and specular spectrals, using Equation (39) and (40). Fig.19 shows the real illumination distribution of outdoor and incandescent light at $2800 \mathrm{~K}$ and the estimated illumination distributions using diffuse and specular separation algorithm, using red, green, and blue points' diffuse and specular spectrals, using Equation (45) and (46). As can be observed in Fig.19, utilizing diffuse and specular points using Equation (45) and (46) produces excellent results.

Table. 1 shows the error value of each algorithm, by using multi point alogrithm, especially using algorithm of Section3.2.2, we have a great performance.

Then, to take a spectral images instead of point spectral distribution, we use a interference filter. We use, SONY XC-55, monocronic camera, and attach a interference filter. Then we make the camera pan rotation and parallel translation. Fig.20 shows a conversion function when a camera take a pararell transformation. This data is obtained by using an algorithm (64). First, we take a Macbeth Chart continuously under blue illumination, moving a camera parallely, and mosaic these images. And compare these data and measured real distribution obtained by using a spectrometer. By using this compensation function, we can obtain a real spectral distribution from a mosaiced images.

Fig. 21 shows a scene separation result using algorithm (26). Take a scene moving a camera parallel translation under incandescent light at $2800 \mathrm{~K}$ and outdoor illumination. Upper image is a spectral image under each light source, center 
graph is separation results of illumination and lower image is estimated surface reflectance. It has some good result in separation, but there are some badness.

Fig. 22 shows a scene separation result using specular and diffuse point. Upper left shows an RGB image that is changed from spectral distribution. Each pixels has a spectral data like a upper right. Spectral signal was obtained using interference filter systems moving a monocronic camera pan rotated under incandesent lightsource. Upper right shows obtained specular and diffuse spectral distribution. The image position of the data is square blue center of upper left image. Lower right shows a real illumination spectral distribution and the result of separation algorithm of (47). It shows a good performans. And lower left is a reflectance images. That is make surface spectral reflectance visualize. For this experiment it is most important to make a great mosaicing because if specular position is miss matched, the specular data become to include a bad noise. It is difficult to remove. But if we get a exact specular data, we can have a so good separation results. 

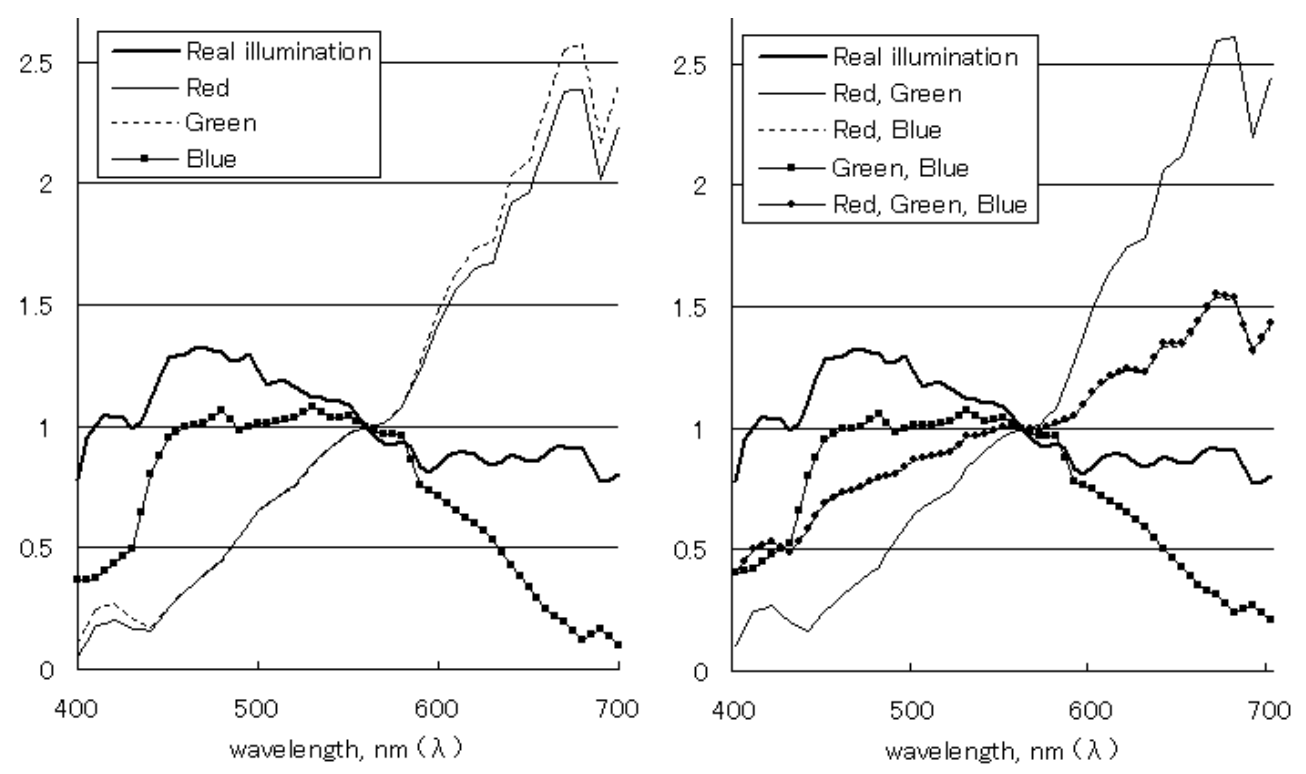

Figure 13: Measured real illumination distribution of outdoors and the result of separation algorithm using left: one point and right: multi points.
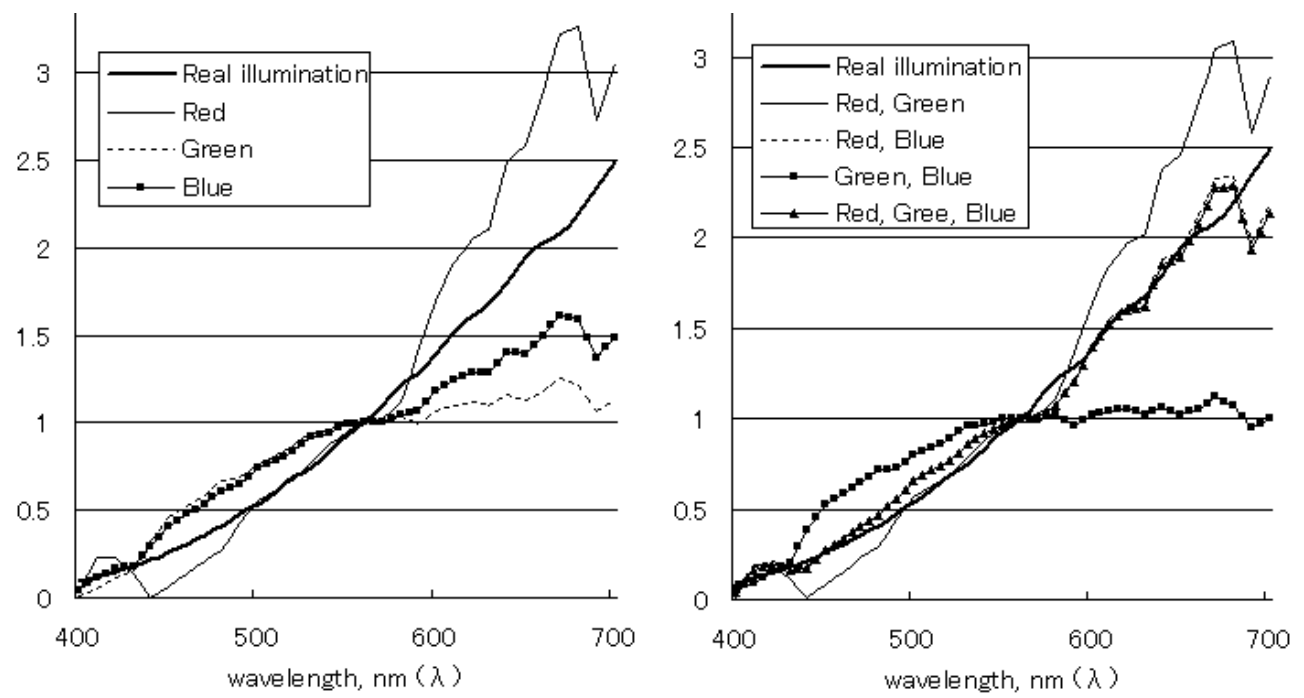

Figure 14: Measured real illumination distribution of incandescent light at $2800 \mathrm{~K}$ and the result of separation algorithm using left: one point and right: multi points. 


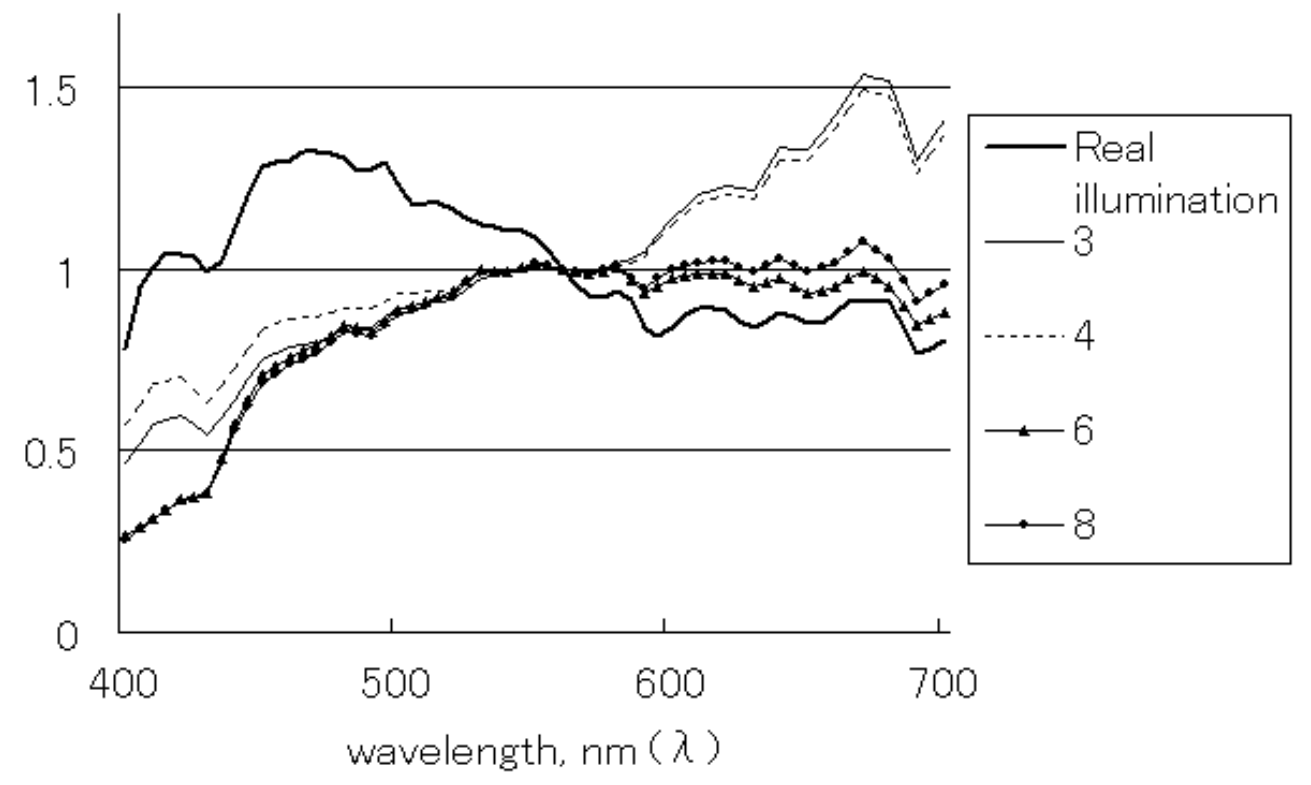

Figure 15: Measured real illumination distribution of outdoors and result of separation algorithm with three, four, six and eight reflectance basis functions using all eighteen colors of the Macbeth Color Checker. 

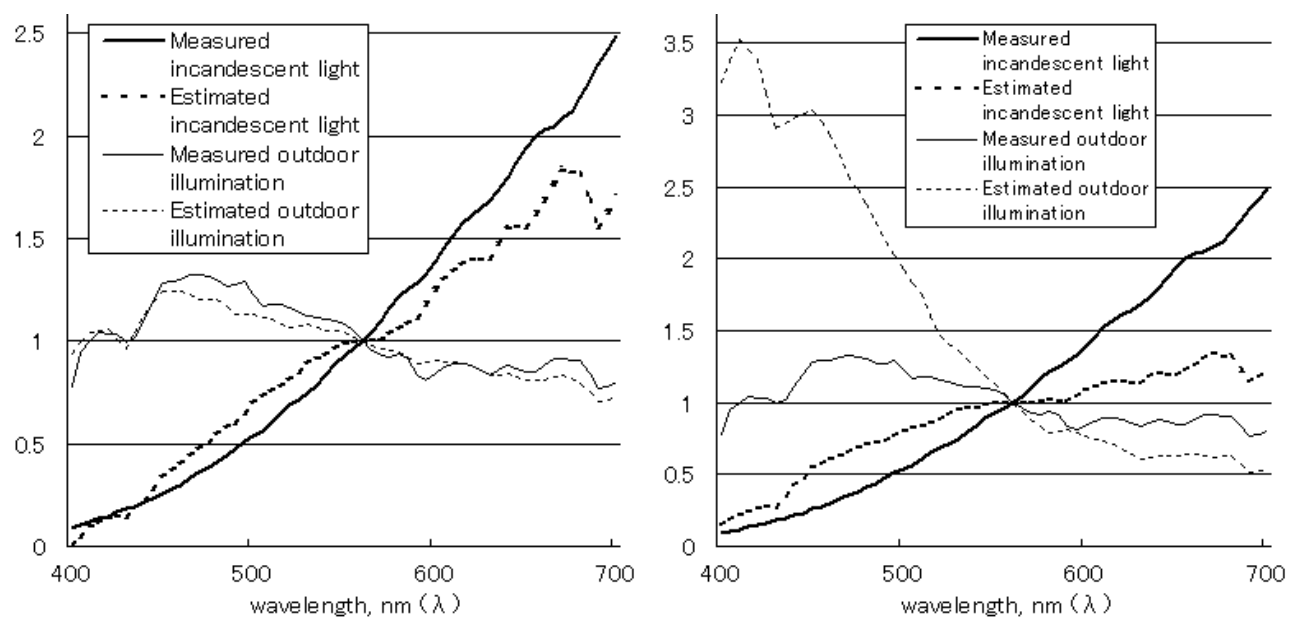

Figure 16: Measured real illumination distribution of outdoor and incandescent light at $2800 \mathrm{~K}$ and the result of the separation algorithm using identical surface(left:blue, right:green) reflectance under these illuminations. 

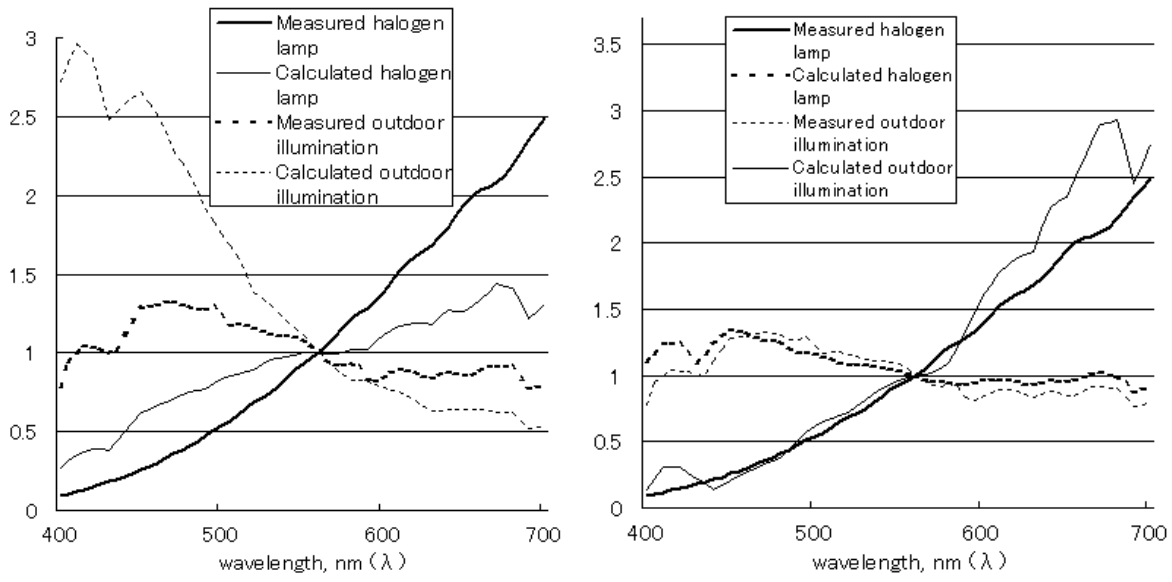

Figure 17: Measured real illumination distribution of outdoor and incandescent light at $2800 \mathrm{~K}$ and the result of the separation algorithm(25) using identical surface(left:blue, right:red) reflectance under these illuminations. 

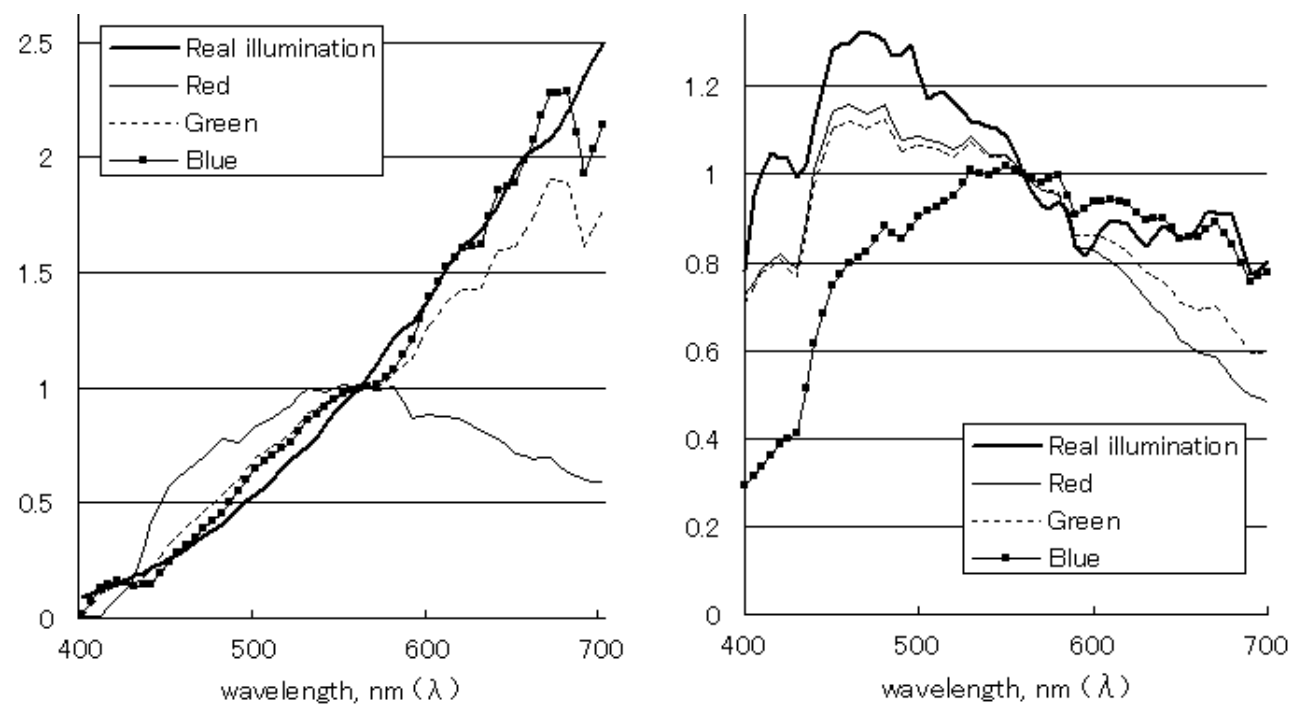

Figure 18: Measured real illumination distribution of left: halogen lamp right:outdoors, and the result of separation algorithm, Equation (39) and (40), using red, green, and blue diffuse-specular points. 

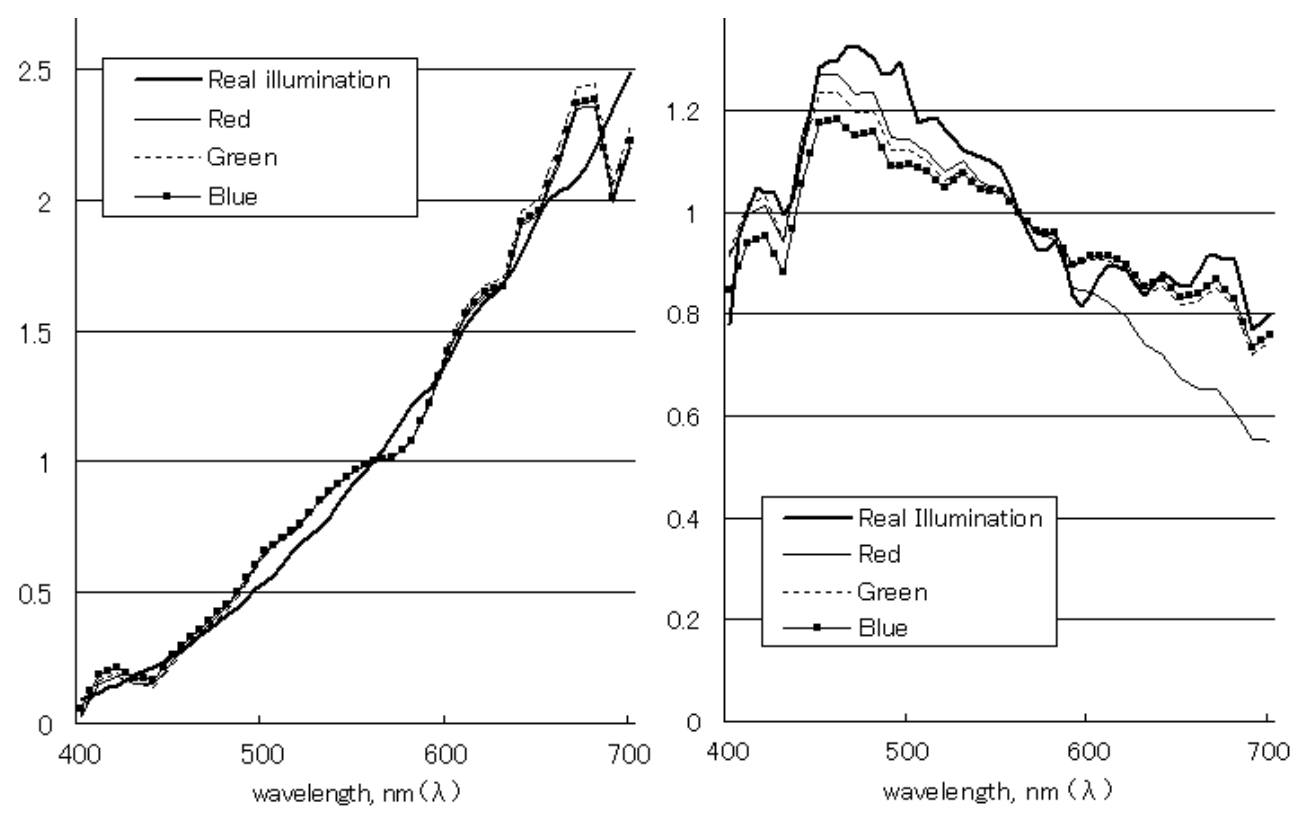

Figure 19: Measured real illumination distribution of left: halogen lamp and right:outdoor, and the result of separation algorithm, Equation (45) and (46), using red, green, and blue diffuse-specular points. 


\begin{tabular}{|l|c|c|c|c|c|}
\hline light source & one point & S.3.1 & S.3.2 & S.3.3.1 & S.3.3.2 \\
\hline blue & 34.55 & 11.39 & 28.10 & 13.01 & 0.76 \\
\hline red & 11.22 & 0.79 & 26.16 & 3.18 & 0.54 \\
\hline
\end{tabular}

Table 1: The error value of one point algorithm and our algorithm(Section number), using all combination of eighteen color of Macbeth Color Checker 


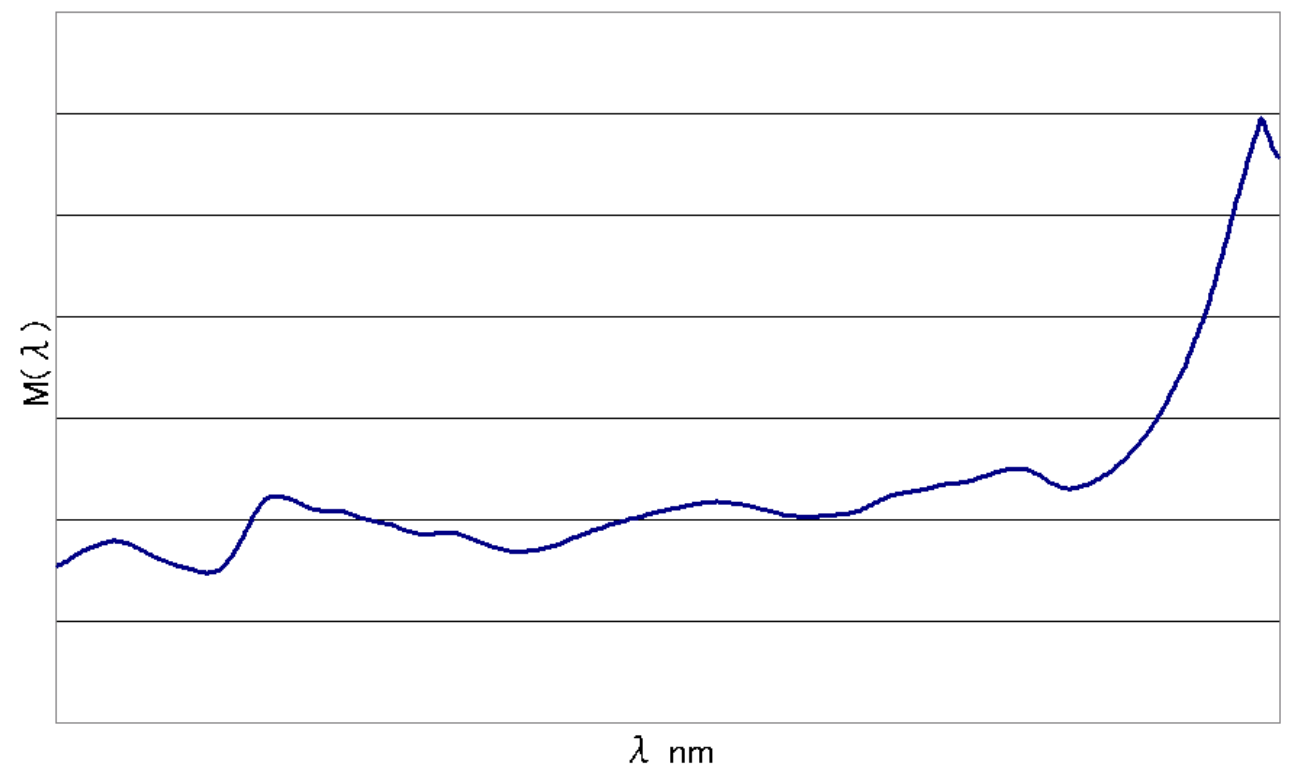

Figure 20: Conversion function of pararell transformation using an algorithm (64). 


\section{Outdoor}
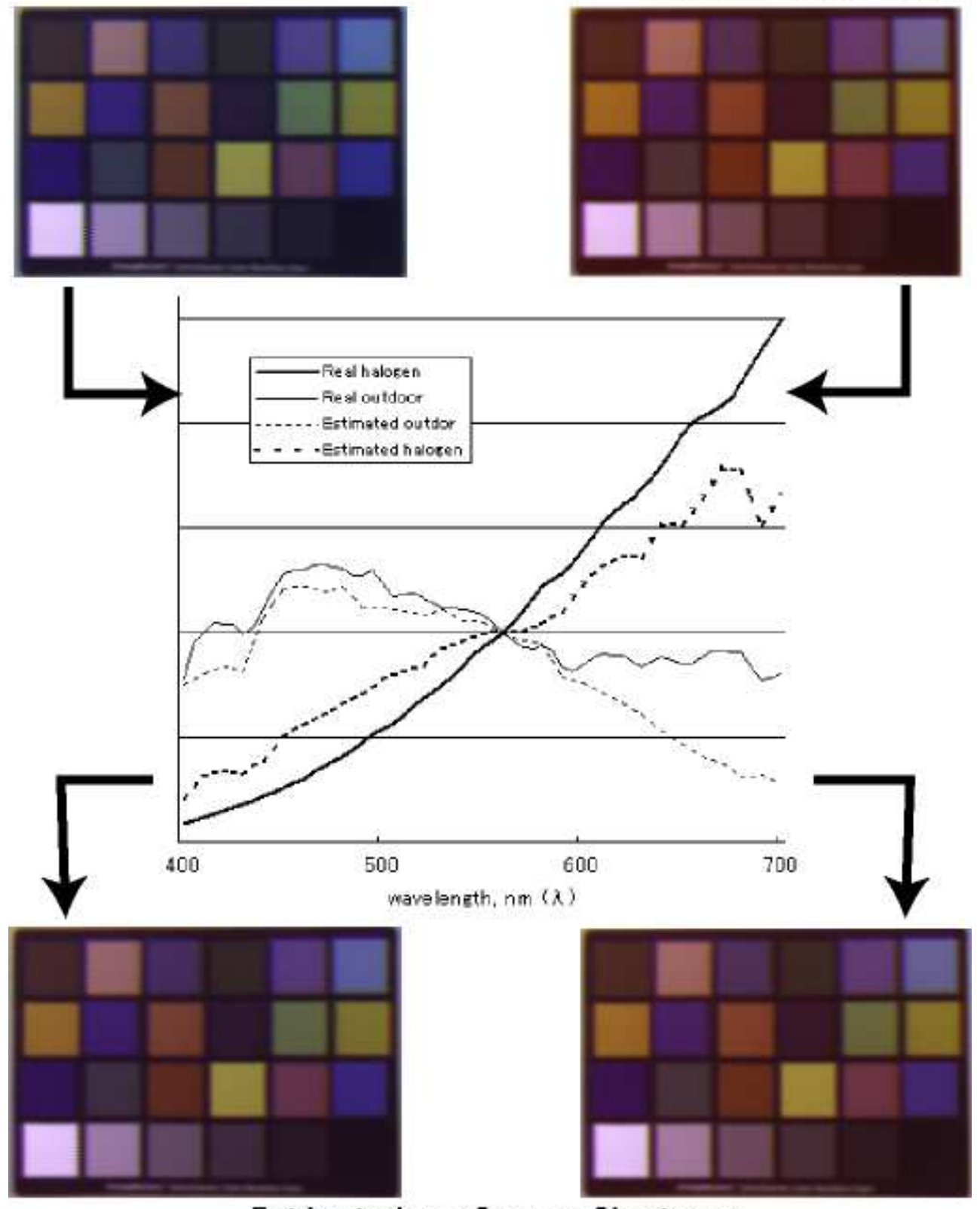

Estimated surface reflectance
Incandescent I ight

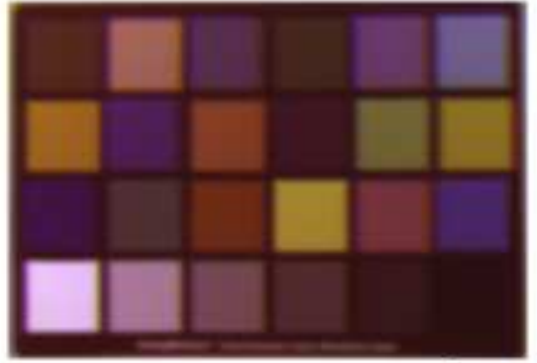




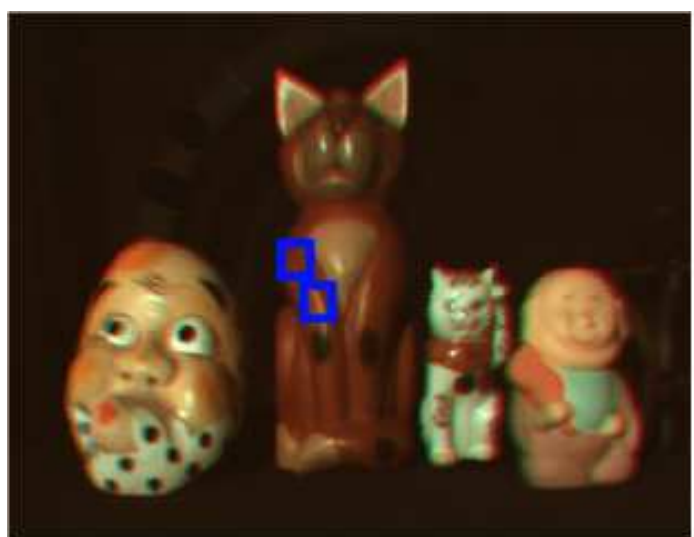

Multi spectral image.

Each point has a spectral distribution.

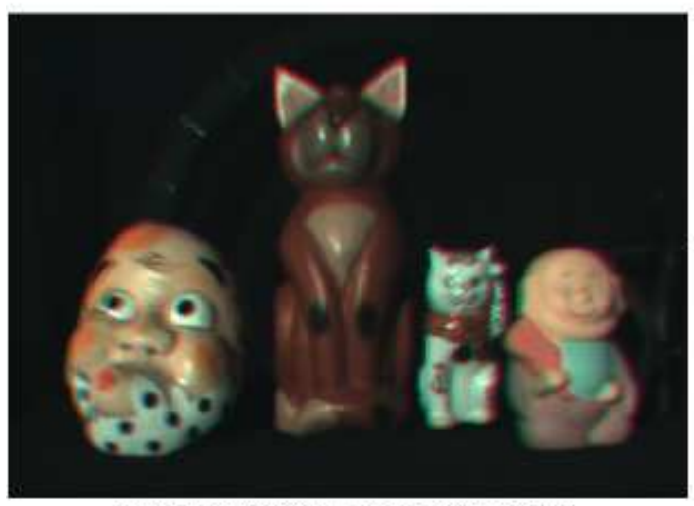

Surface spectral reflectance image. Each point has a spectral reflectance.

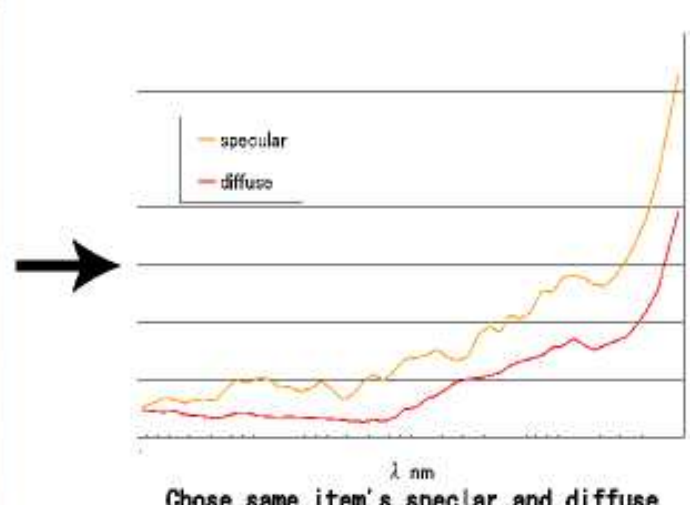

Chose same item's speclar and diffuse spectral distribution.

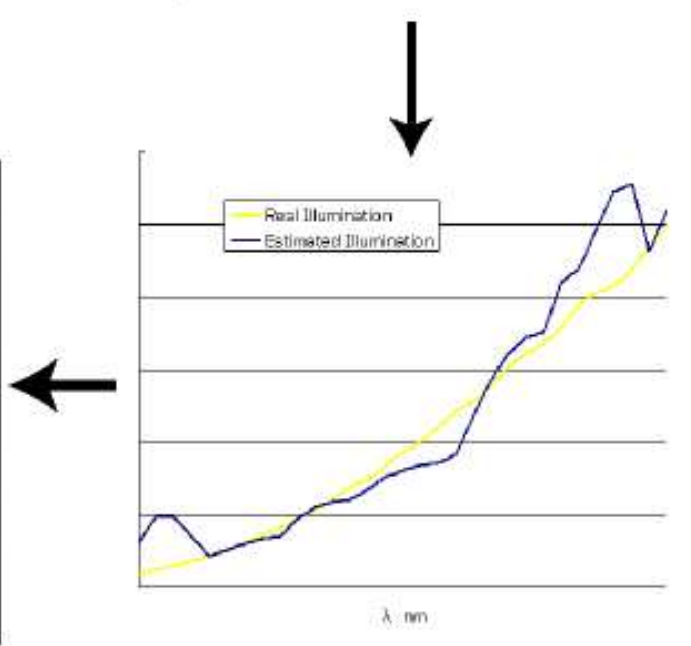

Estimate an illumination distribution.

Figure 22: Scene separation result using specular and diffuse point. Take a scene moving a monocronic camera pan rotated under outdoor illumination. 


\section{Conclusion}

In this paper, we have evaluated a spectrum separation method under three different conditions, including 1) points with different color spectrum distributions illuminated by the same light source, 2) the same point under different illumination sources of different spectrum color distributions, 3) diffuse and specular points under the same illumination source. Using multi point spectrum distributions reliability in the separation of observed scene spectrum distribution into illumination and reflectance spectrum distribution increases. And we have a good result in separating a spectral which obtained using interference filter into illumination spectral distribution and surface spectral reflectance. 


\section{References}

[1] Po-Rong Chang and Tsung-Hsieh Hsieh. Constrained nonlinear optimization approaches to color-signal separation. IEEE trans. image processing, 4(1):81-93, 1995.

[2] J. Cohen. Dependency of the spectral reflectance curves of Munsell color chips. Psychon. Sci., 1:369-370, 1964.

[3] M. D'Zmura and P. Lennie. Mechanisms of color constancy. J. Opt. Soc. Am. A, 3(10):1662-1672, 1986.

[4] Michael D'Zmura. Color constancy: surface color from changing illumination. JOSA, 1992.

[5] G.D. Finlayson, B.V. Funt, and K. Barnard. Color constancy under varying illumination. ICCV, pages 720-725, 1995.

[6] G.D. Finlayson, Steven D. Hordley, and Paul M. Hubel. Color by correlation: A simple, unifying framework for color constancy. PAMI, 23(11):1209$1221,2001$.

[7] G.D. Finlayson and Gerald Schaefer. Solving for colour constancy using a constrained dichromatic reflection model. IJCV, 42(3):127-144, 2001.

[8] Graham D. Finlayson and Steven D. Hordley. Color constancy at a pixel. J. Opt. Soc. Am. A, 18(2):253-264, 2001.

[9] Th. Gevers, H.M.G.Stockman, and J.van de Weijer. Color constancy from hyper-spectral data. $B M V C, 2000$.

[10] Jian Ho, Brian V. Funt, and Mark S. Drew. Separating a color signal into illumination and surface reflectance components: Theory and applications. IEEE PAMI, 12(10):966-977, 1990. 
[11] Deane B. Judd, David L. Macadam, and Gunter Wyszecki. Spectral distribution of typical daylight as a function of correlated color temperature. $J$. Opt. Soc. Am, 54(8):1031-1040, 1964.

[12] H. C. Lee, E. J. Breneman, and C. P. Schulte. Modeling light reflection for computer color vision. PAMI, 12:402-409, 1990.

[13] John A. Marchant and Christine M. Onyango. Spectral invariance under daylight illumination changes. J. Opt. Soc. Am. A, 19(5):840-848, 2002.

[14] Yuu Nakatani. Image mosaicing using active camera. The Institute of Image Electronics Engineers of Japan, 29(5), 2000.

[15] Michiko Minoh-Masashi Yasuda Naoki Chiba, Hiroshi Kano. Feature-based image mosaicing. The Institute of Electronics, Information and Communication Engineers, 82:1581-1589, 1999.

[16] J. P. S. Parkkinen, J. Hallikainen, and T. Jaaskelainen. Characteristic spectra of Munsell colors. J. Opt. Soc. Am. A, 6(2):318-322, 1989.

[17] Markku Hauta-Kasari Reiner Lenz, Peter Meer. Spectral-based illumination estimation and color correction. CCC, pages 98-111.

[18] Yoav Y. Schechner and Shree K. Nayar. Generalized mosaicing: Wide field of view multispectral imaging. PAMI, 2001.

[19] David Slater and Glenn Healey. What is the spectral dimensionality of illumination functions in outdoor scenes? CVPR, pages 105-110, 1998.

[20] Richard Szeliski and Hung-Yeung Shum. Creating full view panoramic image mosaics and environment maps. SIGGRAPH, pages 251-258, 1997. 
[21] Robby T. Tan, Ko Nishino, and Katsushi Ikeuchi. Illumination chromaticity estimation using inverse-intensity chromaticity space. CVPR, 1:673-680, 2003.

[22] Shoji Tominaga. Multichannel vision system for estimating surface and illumination functions. J. Opt. Soc. Am. A, 13(11):2163-2173, 1996.

[23] Shoji Tominaga, Satoru Ebisui, and Brian A. Wandell. Scene illuminant classification: brighter is better. J. Opt. Soc. Am. A, 18(1):55-64, 2001.

[24] Shoji Tominaga and IEEE Member. Surface identification using the dichromatic reflection model. PAMI, 13(7):658-670, 1991.

[25] Shoji Tominaga and Brian A. Wandell. Standard surface-reflectance model and illuminant estimation. J. Opt. Soc. Am. A, 6(4):576-584, 1989.

[26] Shoji Tominaga and Brian A. Wandell. Natural scene-illuminant estimation using the sensor correlation. PROCEEDINGS OF THE IEEE, 90(1):42-56, 2002.

[27] Yalin Xiong and Ken Turkowski. Registration, calibration and blending in creating high quality panoramas. Fourth IEEE Workshop on Applications of Computer Vision, pages 69-74, 1998.

[28] Jon Y.Hardeberg. On the spectral dimensionality of object colours. CGIV, pages 480-485, 2002. 